\title{
Einstein's Dark Energy via Similarity Equivalence, 'tHooft Dimensional Regularization and Lie Symmetry Groups
}

\author{
Mohamed S. El Naschie \\ Department of Physics, Faculty of Science, University of Alexandria, Egypt \\ Email: Chaossf@aol.com
}

Received 25 February 2016; accepted 19 March 2016; published 22 March 2016

Copyright (C) 2016 by author and Scientific Research Publishing Inc.

This work is licensed under the Creative Commons Attribution International License (CC BY). http://creativecommons.org/licenses/by/4.0/

(c) (i) Open Access

\section{Abstract}

Realizing the physical reality of 'tHooft's self similar and dimensionaly regularized fractal-like spacetime as well as being inspired by a note worthy anecdote involving the great mathematician of Alexandria, Pythagoras and the larger than life man of theoretical physics Einstein, we utilize some deep mathematical connections between equivalence classes of equivalence relations and E-infinity theory quotient space. We started from the basic principles of self similarity which came to prominence in science with the advent of the modern theory of nonlinear dynamical systems, deterministic chaos and fractals. This fundamental logico-mathematical thread related to partially ordered sets is then applied to show how the classical Newton's kinetic energy $E=1 / 2 m v^{2}$ leads to Einstein's celebrated maximal energy equation $E=m c^{2}$ and how in turn this can be dissected into the ordinary energy density $E(O)=m c^{2} / 22$ and the dark energy density $E(D)=m c^{2}(21 / 22)$ of the cosmos where $m$ is the mass; $v$ is the velocity and $c$ is the speed of light. The important role of the exceptional Lie symmetry groups and 'tHooft-Veltman-Wilson dimensional regularization in fractal spacetime played in the above is also highlighted. The author hopes that the unusual character of the analysis and presentation of the present work may be taken in a positive vein as seriously attempting to propose a different and new way of doing theoretical physics by treating number theory, set theory, group theory, experimental physics as well as conventional theoretical physics on the same footing and letting all these diverse tools lead us to the answer of fundamental questions without fear of being labelled in one way or another.

\section{Keywords}

Equivalence Relation, Scaling, Intermediate Asymptotic, Golden Mean Scaling, Einstein Self Similarity, Fractal Scaling, E-Infinity, Special Relativity, Random Cantor Sets, 'tHooft Regularization, Fractal Quantum Field, Quantum Gravity, Exceptional Lie Symmetry Groups 


\section{The Simplexity of Quantum Complexity-An Informal Introduction}

The present paper was initially motivated by the wider implications of a small discovery attributed to a young Albert Einstein and the imagined wider implications for his later work on his famous formula $E=m c^{2}$ which we allege could have taken place. However the main thrust of the paper is to demonstrate in a concrete way a general methodology or maybe a new theory in theoretical physics which we could have labelled "Simplixity method of quantum complexity via topological E-infinity theory". To explain as briefly as possible what we mean by that, it is best to start with a concise resume of basic theories connecting self similarity, fractals and physics [1]-[40].

In mathematics, it is well known that not only Cantorian fractals probabilistic equivalence relation (see Appendixs 1-4) can be based on similarities and we will show here how this can lead to surprizing connections to physics via Penrose fractal tiling based self similar E-infinity quotient spacetime manifold [1]-[20]. Self similarity may be seen as trivially obvious as the number ten, being ten copies of unity and/or as quite complex as the Julia and Mandelbrot set of fractal dynamics [1]-[6]. Self similarity (see Appendices 1-3) is in the meantime a well known fundamental principle of modern nonlinear dynamics, theory of fractals (see Figures 1-3) as well as deterministic and quantum chaos in physics and cosmology [7]-[18]. More recently fractal-Cantorian spacetime and E-infinity theory demonstrated how self similarity is intimately and closely related to the renormalization procedures [19]-[25] of quantum field theory and 'tHooft's dimensional regularization [26]. In fact the said E-infinity Cantorian spacetime theory [26]-[34] is built almost entirely on the explicit self similarity of the expectation value of its Hausdorff dimension [10] [28]

$$
D=4+\phi^{3}=4+\frac{1}{4+\frac{1}{4+\cdots}}
$$

where $\phi=(\sqrt{5}-1) / 2$ [7]-[17]. It should therefore be expected as a matter of logical consistency that the equation derived from and in this spacetime will also be self similar or at a minimum self affine [23]-[45]. This is actually the main theme of the present paper [1]-[106] and at the end of a rather detailed discussion and analysis we will rediscover a fundamental fact, namely that most of the experimentally verified theories were always self

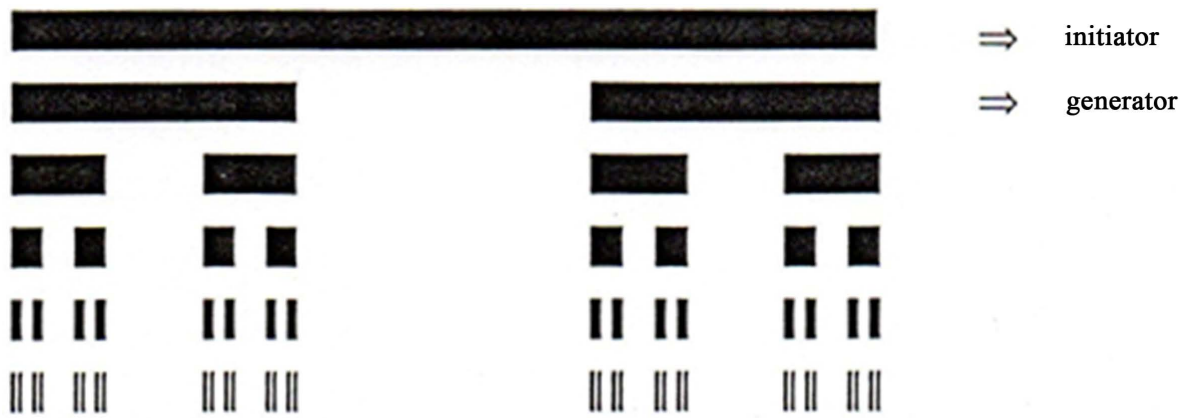

Figure 1. A one dimensional Cantor universe (see Refs. [1] [2] and [6]).

1

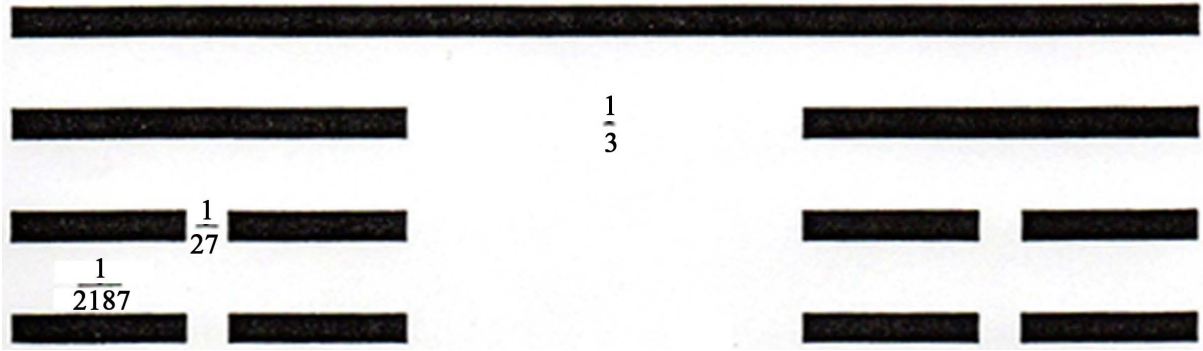

Figure 2. Construction of a fat fractal. It has infinitely many holes but the remainders keep a total length that is greater than zero (see Ref. [6]). 


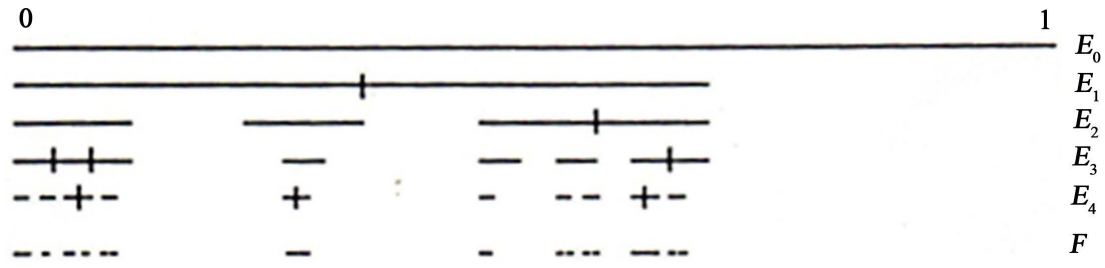

(a)

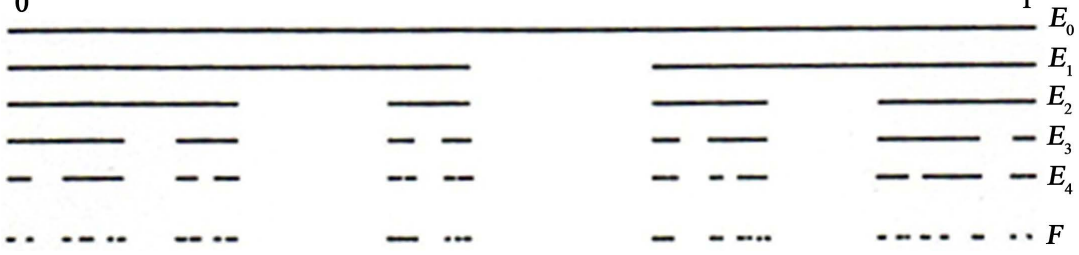

(b)

Figure 3. (a) and (b) - Two random versions of the Cantor set. In (a) each interval is divided into three equal parts from which some are selected at random. In (b) each interval is replaced by two subintervals of random lengths.

similar in one way or another [106]. Looking for instance at fields as diverse as classical gravity [17] [24] [30], electric fields as well as magnetic fields we find that the basic inverse square law reigns supreme [46]-[48]. In fact and as will be analysed in substantial detail in the present work, Newton's kinetic energy $E=1 / 2 m v^{2}$ where $m$ is the mass and $\mathrm{v}$ is the velocity and probably the most famous formula in physics, with which we mean Einstein's mass-energy equation $E=m c^{2}$ where $c$ is the speed of light (see Figure 3) differ only formally in the scale and notation used and nothing much more than that (see Figure 3 and Figure 4). Remembering that energy, entropy and information [46] [49]-[57] are without a doubt some of the most fundamental and interrelated notions in physics, then it is natural that we utilize these afore mentioned self similarity to shed light on the major problem of the measurable ordinary energy density [58]-[63] and the dark energy density [60]-[63] of the cosmos which we cannot measure at present in any direct way [57]-[63]. As mentioned earlier on, this and various related aspects [60]-[74] is the subject of the present paper which although relatively short, has the character of a survey paper due to the large number of subjects and problems we address as well as the numerous theoretical and mathematical physics references included [1]-[132]. On the other hand we give limited space to the pure mathematical literature of equivalence relations which is never the less vital to an even deeper understanding of our method and results. This short coming could be helped by consulting Refs. [105]-[111] (see also Appendix 4). The author would like to apologise for several quite unavoidable gaps in the presentation which he tried hard to minimize in the included 8 appendices. In this respect we stress that reading Refs. 121 to 135 is strongly recommended to gain a deep insight into the subject.

\section{Fuzziness as a Method}

The title of this section is not in praise of being sloppy in physics but simply a somewhat provocative invitation to take fuzzy logic [11], fuzzy sets [76]-[85] and consequently Cantor sets, fractals and transfinite set theory very seriously in quantum physics [70]-[106]. Nature is overwhelmingly fuzzy in an irreducible and fundamental way as reflected in the mathematics of even the most orthodox quantum mechanics theories, Hardy's quantum entanglement included [54]. Consequently to force nature to be described mathematically in a sharp, crisp way is really forcing it to submit to an inaccurate mathematical formulation [11]. As an example of what we have in mind, let us count quantum particles. At the beginning there was SU(3) SU(2) and U(1) of the standard model [13] [28] [46]. The number of the generators of this combined Lie symmetry group corresponds to a dimension equal to $8+3+1=12$ and this number corresponds, as is well known, to eight gluons, three massive photons and one massless ordinary photon [47]. So are quantum particle isometries of the same Lie symmetry spaces corresponding to Lie symmetry groups - this may be a naïve question not worthy of a question mark. In fact nature is far stranger than fiction because fractal logic [83] teaches us that these 12 messenger particles are in reality 14 particles and have the fractal weight number of 11.708239325 [83]. Now regardless how we answer 


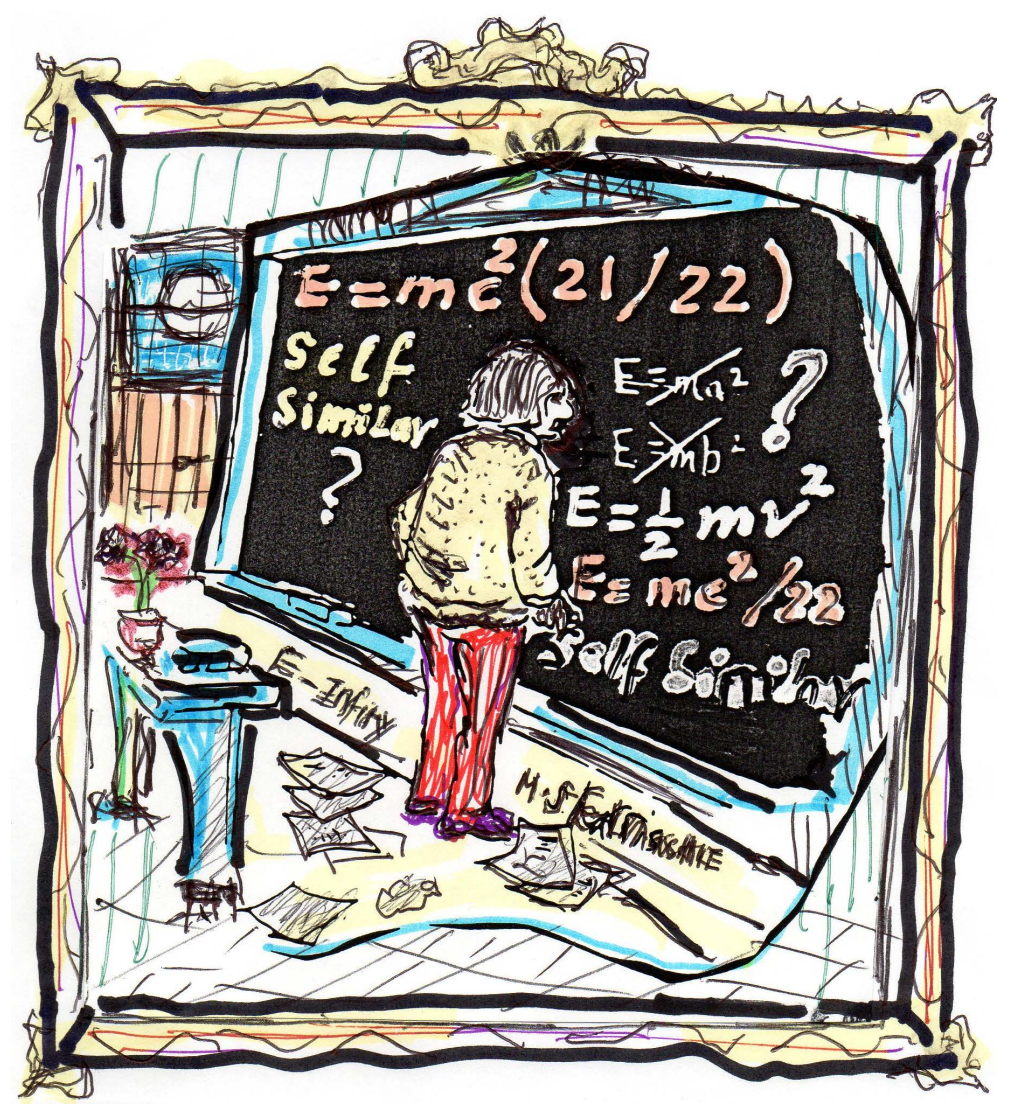

Figure 4. A light hearted, not historical picture depicting the moment when the truly great Albert Einstein was about to discover E-infinity dark energy formula $E(D)=m c^{2}(21 / 22)$ as well as the ordinary energy formula of the same theory $E(O)=m c^{2} / 22$. It seems possible that after giving an alternative proof for the theorem of Pythagoras based on the principle of self similarity that he realized that this theory and Newton's kinetic energy $E=1 / 2 m v^{2}$ are self similar. That way he could have discovered the chaotic nonlinear dynamics beneath quantum mechanics and found El Naschie's dissection of $E=m c^{2}$ into its quantum components $E=\left(m c^{2} / 22\right)+m c^{2}(21 / 22)$ (see Refs. [36] and [50]).

this naïve question and the puzzling fractal logical reality, we hasten to say that E8E8 super string theory starts with 496 quasi massless gauge bosons and not 12 and one has normally to show how these 496 isometries are reduced via symmetry breaking to our observed 12 gauge bosons of the standard model [28] [47]. In addition we know from bosonic string theory that spacetime is 26 dimensional and when we imagine 16 dimensions running in an opposite direction to these 26 , then we find $26-16=10$ which corresponds to one type of a so called heterotic super string theory [28] [47] of which one may be based on E8E8 or on SO(32) which leads to [101][105] [124]-[126]

$$
|S O(32)|=(32)(32-1) / 2=496
$$

as in the $E 8 E 8$ where $E 8$ is the largest exceptional Lie symmetry group [28] [55] [56]. Thus we could really think of internal space dimensions as particles resulting from symmetry breaking related to Noether's conservation laws and symmetry theorem and if we venture into even more "fuzzy" spacetime dimensions [41] such as $D$ $=11$ of Witten, $D=5$ of Kaluza-Klein then even the $D=4$ of Einstein could be seen as a kind of pre-particles [47]. But what good could this wild mixing of basically different notions and concepts bring about except a little more chaos added to a mess of other problems? We could answer this point by working out something specific, namely determining the density of ordinary energy, dark energy, dark matter energy and pure dark energy density of the cosmos. This is what we will do next [63]-[77].

The standard model contains 126 particle-like states when considering super symmetry and disregarding frac- 
tal logic counting [28] [46]. In the case of super gravity this contain 2 more, namely 128 which corresponds to half of the 256 Einstein-Riemann independent curvature tensor components as given by $(4)^{4}=256$ [48]. Without super symmetric partners this corresponds to 64 particles in $4 \mathrm{D}$, which we can detect only if we perform experiments in 4D. However, real experiments can be done only in 3D with time as a parameter [47].

Taking our previous discussion into account as well as realizing that all energies must ultimately be a scaling of Einstein's maximal energy $E=m c^{2}$, we are inclined to conclude that a good estimate of the ordinary, real measurable energy density of the universe must be $E=m c^{2}$ scaled by the ratio of real space $D=3$ to the space of the standard model plus gravity which is in the afore mentioned fuzzy meaning $D=64$ so that one finds approximately [36]-[45]

$$
E(O) \simeq\left(\frac{3}{64}\right)\left(m c^{2}\right) \simeq 0.046875 m c^{2} \simeq 4.7 \%
$$

of the total Einstein energy in reasonable agreement with the cosmic measurements [36] [47]. To account for dark energy we have the self explanatory scaling $\lambda \cong(64-3) / 64=95.312 \%$, again in fair agreement with measurements [37]-[45]. Finally to find the scaling associated with dark matter, we see that it is reasonable to consider the ratio of the total number of the 12 messenger particles of the standard model plus the Higgs and the graviton making them 14 particles corresponding to the dimension of $\left|G_{2}\right|=14$ exceptional Lie groups or dim OSP $(1 / 4)=14$ of orthosimplectic group [28], for which one finds

$$
D(\text { dark matter }) \simeq\left(\frac{14}{64}\right)\left(m c^{2}\right) \simeq 21.875 \%
$$

Yet, again in fair agreement with the limit which cosmic measurements set on the dark matter energy density [36]-[47] [60]. In Appendix 8 a preliminary, simple and intuitive derivation of the above is given using a radically different conception.

\section{A Thin and a Fat Cantor Universe Made of One Cantor Set and Its Embedding}

Following the pictorial logic of the one dimensionally embedded iterative triadic Cantor set of Figure 1 we see that it leads us to consider two sets (see also Appendix 1). The first is the classical triadic set consisting of an uncountable infinite number of black Cantor points with a Hausdorff dimension equal $D(H)=\ell n 2 / \ell n 3 \simeq 0.63$ and a topological dimension equal zero and in addition the length of this set is zero, i.e. it is a zero measure geometrical-topological structure. This is basically the zero set which models in E-infinity theory the pre-quantum particle. The second set is the white gaps between the black Cantorian points of the zero set [36]-[45]. The Hausdorff dimension of this set is simply one minus the Hausdorff dimension of the zero (black) Cantor set, i.e. $1-0.63 \simeq 0.37$. The measure of this set on the other hand is equal to one minus zero which is then equal one indicating a fat Cantor set as shown in Figure 2 [72]. Since this set represents the gaps in the "black" set, it is essentially an empty set with a negative topological empty set dimension equal minus one. It follows then that this "gaps" Cantor set is the empty set which models the pre-quantum wave in E-infinity theory [10] [13] [28]. The situation remains the same for a random Cantor set (see Figure 3) except that $\mathrm{D}(\mathrm{H})$ goes to $\phi^{2}=(\sqrt{5}-1) / 2$ and thus $1-D(H)$ goes to $\phi^{2}=0.381966$ instead of 0.37 [36]-[63].

It follows then from the geometry and topology of the above that each black point is essentially a pre-quantum particle with a positive topological attracting pressure equal to the Hausdorff dimension of the zero set which is $\phi$ while the white gap between them is the empty set modelling the pre-quantum wave with an opposing topological pressure equal to the Hausdorff of the empty set, namely $\phi^{2}$ [62]-[76]. Consequently we have a resulting net pressure equal $\phi-\phi^{2}=\phi^{3}$ which we can view as the topological local Casimir force. In turn $\phi^{3}$ is equal to the Hausdorff dimension of the cobordism [35] [42], i.e. the surface of the quantum wave as well as being the expectation value of the multi-fractal surrounding of the quantum wave giving rise to the core of quantum spacetime as is obvious from the relation [63]-[76]

$$
d_{c}^{(4)}=(1 / \phi)^{4-1}=1 / \phi^{3}=(1 / \phi)^{3}=4+\phi^{3}
$$

where $D(T)=4$ is the topological dimension and $D(H)=4+\phi^{3}$ is the expectation Hausdorff dimension of 
quantum spacetime. We conclude that the above is an accurate one dimensional Cantorian spacetime which could now be expanded to the fully fledged 5 dimensional Kaluza-Klein fractal spacetime of our reality [63]-[76]. That way the pressure $\phi^{2}$ behind the local Casimir pressure $\phi-\phi^{2}=\phi^{3}$ becomes a five dimensional union $\phi^{2}$ empty sets equal (5)( $\left.\phi^{2}\right)$ which leads to a dark energy density equal to [63]-[76]

$$
E(D)=\left(5 \phi^{2}\right)\left(\frac{1}{2} m\right)(v \rightarrow c)^{2}=\left(5 \phi^{2} / 2\right) m c^{2}
$$

This is self evidently the exact dark energy density found via accurate measurement and cosmological observation. Our second most import conclusion is that the Casimir energy is the local form of dark energy which by the well known theorem of Dvoretzky must be concentrated at the boundary of the holographic boundary of the universe [76]. This is one of the reasons why it cannot be detected locally in direct experiments although we know of its presence directly via the observed accelerated cosmic expansion [37]-[49]. Related similar results connected to formal logic and self referentiality is considered in [117]. We stress that the above results agree with the basic features of the excellent analysis of Mageuijo [128] and Smolin [127].

\section{The Double Nature of a Cantor Set in Mathematics and Physics}

It is an illusion to think that a Cantor set is intuitively simple. A Cantor set is in fact both the simplest and the most mind boggling thing that there is on the foundational level of pure mathematics and mathematical physics.

To start with a Cantor set construction produces at the end two contradictory Cantor sets. Let us look at the classical iterative procedure of Figures 1-3 which is rather familiar to those working in any of the many branches of nonlinear dynamics, chaos and fractals [1]-[5] [64]-[76]. At the final stage after a theoretically infinite number of iterations we end up with an uncountable infinite number of black Cantorian "points" separated by an equally uncountable infinite number of white gaps. For the black set we have a truly contradictory existence because the length of this set is clearly zero, i.e. it is "measure" zero and consequently in the limit it is not there in any physical sense yet this set which is zero topological dimensions, has another substantial dimension, namely a Hausdorff dimension equal $\ell n 2 / \ell n 3 \simeq 0.63$. For a randomly constructed Mauldin-Williams Cantor $\mathrm{S}$

[10] the situation is not different where we find that the Hausdorff dimension is equal $\phi=(\sqrt{5}-1) / 2=0.618$

which is not much smaller than the 0.63 of the classical Cantor triadic set [10]. In defence of the convoluted logic of this situation, one may argue that this is a Hausdorff dimension and more related to information and entropy while the familiar topological dimension of this Cantor set is following the Menger-Urysohn dimension theory is really zero. This argument however would not reduce the situation to a more intuitive level but would in fact increase the non-intuitive character of a Cantor set [1]-[10]. In this case we could still be persistent and point out that there is nothing called Cantor points because it is just a matter of scale and when a so called Cantorian point is magnified, we retrieve yet again an entire Cantor set. That would then make some sense because we can deny that there will ever be a real moment when a Cantor set disappears except in our imagined mental picture at infinity, i.e. at a tomorrow while we know that this "domani" will truly never come! But then here comes the next outrageous point related to the white set which compared to the black set is a fat Cantor set because it has a positive measure. Let us pause a moment and recall that we started our Cantor sets construction by a unit interval represented by a line of a topological and Hausdorff dimension coinciding and equal to unity. Similarly the length of this line is also unity. Now to construct the black set we must subtract the total length of this black set from the $\ell=1$ and since the black set is measure zero [1]-[10], the white set would remain with a length equal unity. On the other hand for the Hausdorff dimension we have to subtract $\phi$ of the black set from unity and find the Hausdorff dimension of the fat white set to be $1-\phi=\phi^{2}$. So far so good, but it remains to find the topological Menger-Urysohn dimension corresponding to $\phi^{2}$ [40]-[45]. The safe way for finding out this quantity is to use the dimensional function of von Neumann and Connes and find to our mild surprise that it does represent again a challenge for classical intuition because the topological dimension corresponding to $\phi^{2}$ Hausdorff dimension is neither zero nor one but rather minus one. Of course naïve expectation would have persuaded us to believe that the topological dimension of the dark set is zero so that we need to subtract zero from the topological dimension of the original "line" interval so that we have the dimension one. However such a conclusion would be mathematically totally incorrect because the dimension one is reserved for a classical line while the white or fat Cantor set is not a classical line for the simple reason that it contains in it infinitely many 
black holes forming our thin black Cantor set. To make a long story short, the resolution of this contradiction is found in the topological concept of neighbourhood. It is a well established topological concept that the neighbourhood of a classical point with zero topological dimensions is an empty set with a minus one topological Menger-Urysohn deductive dimension [10] so that we may conclude that first the fat white Cantor set is an empty set and second this empty set is the cobordism, i.e. the surface of the thin black Cantor set [1]-[10]. The final magnificent outcome shows a totally pathological situation to be generic and at the root of not only mathematics but also physics. Quantum physics and cosmology are full with counter intuitive and paradoxical theoretically as well as experimentally well founded results such as quantum entanglement and dark energy. Nature, as seen within the limitation of mans' brain and logic, seems to be fundamentally pathological. Similar to treating bacterial infections using the same weapon, namely antibiotics, nothing is better suited to deal with the natural pathology of nature than the generic pathology of Cantor sets and multi-fractals which explains the undoubted success of E-infinity theory [10] in explaining what quantum entanglement and dark energy are and why $E=m c^{2}$ is actually the sum of two quantum components, namely $E(O)=\mathrm{mc}^{2} / 22$ for ordinary energy [36][45] and $E(D)=m c^{2}(21 / 22)$ for dark energy so that at the end we have the energy of a quantum particle $\mathrm{E}(\mathrm{O})$ added to the energy of a quantum wave E(D) giving us the maximal E of Einstein (see Figure 4(a) and Figure 4(b)) [36]-[45]

$$
E=E(O)+E(D)=m c^{2} / 22+m c^{2}(21 / 22)=m c^{2}
$$

In this sense $E(O)$ could be regarded as the position or potential energy and $E(D)$ as the kinetic energy while $E=m c^{2}$ is the maximal total energy with the possible interpretation of converting mass to energy, only in theory or also in actual real world, but this is not the point we want to discuss in the present context. To enhance understanding of the role of fractals in the present work and for a deeper understanding of the role of dimensions, the reader is referred to Appendixes 1-3. We should also stress at this point the fundamental role played by Hardy's quantum entanglement [74] in dark energy [54] [61]. In addition the crucial role of the bosonic degrees of freedom of the standard model is outlined in Appendix 8.

\section{The Logarithmic Spiral Connection between Quantum Field Theory and E-Infinity Cantorian Spacetime Theory}

The logarithmic spiral with its self similar geometrical structure creating golden mean rectangles step by step as shown in Figure 5 is a remarkable visual confirmation that E-infinity space, quantum field theory logarithmic scaling and the golden mean scaling of E-infinity theory [6] are most probably deeply connected as careful mathematical analysis would reveal [6]-[10]. This is thus more than an intuitive hunch and is clearly the raison d'etre of the success of standard renormalization equations such as [26]-[34] [86] [90]-[93].

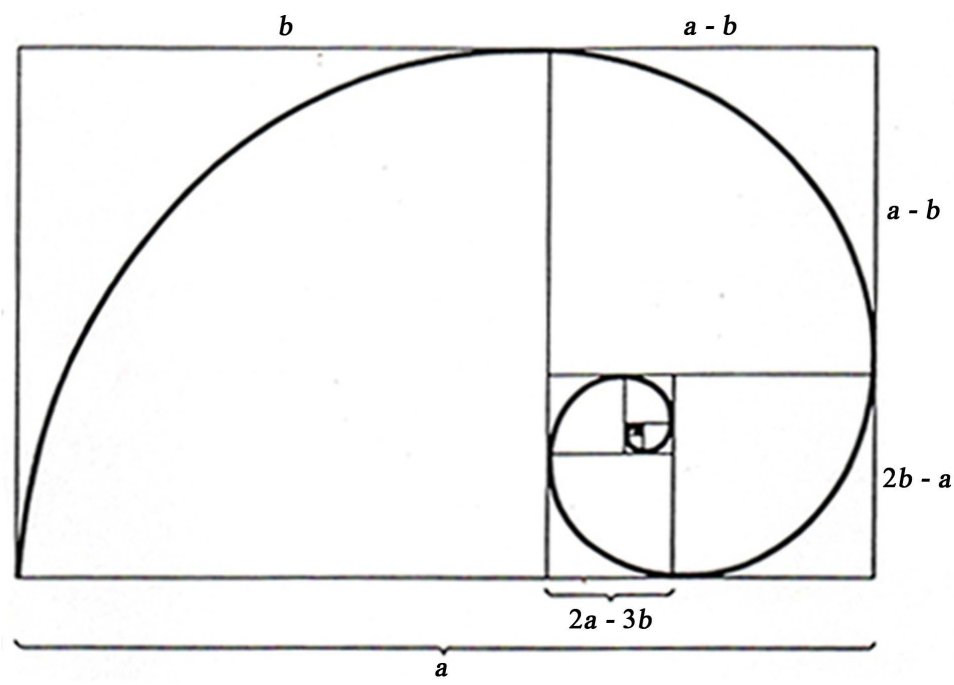


Figure 5. A self similar succession of golden mean rectangles, produced by the logarithmic spiral. This is a clear hint at the deep connection between E-infinity golden mean scaling and the logarithmic scaling of quantum field theory (see Section 5 of the present paper).

$$
\bar{\alpha}_{u}=\bar{\alpha}_{1}+\bar{\alpha}_{2}+\rho \ell n \frac{M u}{M x}
$$

and its exact E-infinity counterpart [90]-[93]

$$
\bar{\alpha}_{u}=\bar{\alpha}_{1}+\bar{\alpha}_{2}+\left(\bar{\alpha}_{o}\right)(\phi)^{n}
$$

This subject is discussed in considerable details in Refs. [26]-[34]. Here we give only the analysis for a grand unification for non-super symmetric $\rho=1$ and the super symmetric $\rho=1 / 2$ case. The unification energy in this case corresponds to a 'tHooft-Polyakovmonopole $M_{u} \cong 10^{16} \mathrm{GeV}$ while the reference mass is taken as usual to be a typical electroweak scale, namely $M_{z}=91 \mathrm{GeV}$. That way we find [88] [90]-[93] [121]-[123]

$$
\bar{\alpha}_{g}=(9+1)+(1) \ln \frac{10^{16} \mathrm{GeV}}{91 \mathrm{GeV}}=10+32.33=42.33
$$

which is very close to the expected accurate value [88]-[91]. For the super symmetric case we find [93]

$$
\bar{\alpha}_{g s}=(9+1)+\left(\frac{1}{2}\right)(32.33)=10+16.165=26.165
$$

Again it is an excellent result compared to the exact one, namely $26+k=26.180339$ so far for the logarithmic scaling of quantum field theory. For the E-infinity golden mean scaling on the other hand we find [86] [91]-[93]

$$
\bar{\alpha}_{g s}=(9+1)+\frac{1}{2}\left(\bar{\alpha}_{o} / 2\right)(\phi)^{2}=(10)+\left[\left(137+k_{o}\right) / 2\right](\phi)^{2}=42.36 \cdots
$$

and [91]-[93]

$$
\bar{\alpha}_{g s}=(10)+\frac{1}{2}\left(\bar{\alpha}_{o} / 2\right)(\phi)^{2}=26+k=26.18033989
$$

exactly as expected [26]-[34]. Note that this is the same result as that which we obtain for quantum gravity [26][34] [86]. In other words when we include super symmetry we automatically include gravity in in our calculation. This becomes clear when we take in the above calculation $M_{u} \cong(10)^{19}$ instead of $(10)^{16} \mathrm{GeV}$ and replace $m_{Z}=91 \mathrm{GeV}$ by that of the electron $m_{e}=0.511 \mathrm{GeV}$. That way we find that [26]-[34] [86] [91]-[93]

$\bar{\alpha}_{3}=0, \bar{\alpha}_{4}=1$ and

$$
\begin{aligned}
& \bar{\alpha}_{u}=1+\frac{1}{2} \ln \frac{10^{19} \mathrm{GeV}}{[(0.511) / 1000] \mathrm{GeV}} \\
& 1+\frac{1}{2} \ln (1.9564)(10)^{22} \\
& 1+\frac{1}{2}(22 \ln 10)+\ln 1.9569 \\
& =1+(51.32) / 2 \\
& =26.6639
\end{aligned}
$$

using the golden mean scaling. On the other hand things are quite straight forward leading to [88] [91]-[93]

$$
\bar{\alpha}_{u}=\left(\bar{\alpha}_{o} / 2\right)(\phi)^{2}=26+k
$$

just as in the grand unification case [91]-[93]. 


\section{No Energy without a Volume}

Clearly there can be no energy without spacetime volume to contain it. This statement remains true even if volume and energy are one and the same thing as in general relativity curvature of spacetime and gravity are one and the same. Now we have two volumes. First we have the volume of the five dimensional pre-quantum particle [26]-[34]. This is a conventional multiplicative volume equal $(\phi)^{5}$ where the 5 is $D=5$ of Kaluza-Klein spacetime [46] and $\phi$ is the Hausdorff dimension of the pre-quantum particle interpreted as a measure [26]-[34]. Second we have the volume of the pre-quantum wave. This is an additive volume being more of the higher dimensional circumferential length of the wave enveloping the particle in $D=5$ which means $5 \phi^{2}$ where $\phi^{2}$ is taken as a measure for the pre-quantum wave. Now since the wave is the surface of the particles, we have as many particles as waves and the particle wave duality leads therefore to the obvious average volume of a quantum particle, namely the arithmetic mean of the multiplicative particle volume and the additive wave volume from which we find [35] [42] [60] [62]

$$
\langle V o l\rangle=\frac{1}{2}\left(\phi^{5}+5 \phi^{2}\right)=\frac{1}{2}(2)=1
$$

Consequently $E=m c^{2}$ could be rewritten as [62] [76]

$$
E=\left(5 \phi^{2}\right) m c^{2}+\frac{5 \phi^{2}}{2} m c^{2}=E(O)+E(D)=m c^{2}
$$

as claimed by us at the very beginning of this section [92]. In the next section we aim to show that the principle of self similarity also holds true for Newton gravity and quantum gravity [61]-[63].

\section{Dark Energy and Ordinary Energy Density of the Cosmos from Dvoretzky's Theorem of Banach Spaces}

There are a few things that we need to know before noticing at once that the Dvoretzky theorem [34] [39] is already the answer to our main question regarding dark energy question. First we must know that Einstein's energy $E=m c^{2}$ is the maximal energy density possible in the universe when $m$ is the mass and c is the speed of light [34] [39]. This formula is simply a similarity gauging of Newton's $E=1 / 2 m v^{2}$ when $1 / 2 \rightarrow 1$ and $v \rightarrow c$. Second we need to reason that a spherical multi-dimensional ball is the most likely shape of our universe. Thus our universe must be at least a five dimensional sphere which may be regarded as a sufficiently high dimensionality to make Dvoretzky's theorem work. Now we see that the theorem employs a volume measure concentration at the surface of the sphere amounting to $96 \%$ while the rest of the universe contains only $4 \%$ of the volume. Since energy must be proportional to the volume of spacetime, it follows directly that $E$ is $96 \%$ at the end of the universe, i.e. at the boundary of the holographic boundary [34]-[39] [42]-[45].

It does not seem farfetched at all that our real spacetime must be at least five dimensional. The 4 dimensions of Einstein are self evidently 3 space and one time dimension which were merely fused together in special and general relativity. On the other hand the phenomenal success of Kaluza-Klein's five dimensional theories, let alone 10 dimensional super strings and eleven dimensional super gravity and Witten's M-theory [47] are all pointing in this direction and seems to reinforce the belief that Dvoretzky's theorem applies indeed to our universe. The measurement of ordinary energy $E(O)=4.5 \%$ and the conjectured dark energy presumed to be the cause for accelerated cosmic expansion consistent with $E(D)=95.5 \%$ with the prediction of Dvoretzky's theorem, namely $E(O)=4 \%$ inside the universe which means local ordinary energy density and dark energy density $E(D)=96 \%$ at the boundary of the universe causing the expansion must be regarded as an excellent experimental confirmation of Dvoretzky's theorem applicability to our cosmic fractal "manifold" [42]. We could give a rather short and neat theoretical analysis confirm the above without appealing in any direct or indirect way to Dvoretzky's theory. To do this we start from E-infinity theory [34] [39] at the observable electroweak energy scale for which we have [88] [91]-[93]

$$
\bar{\alpha}_{o}=(1 / \phi) \bar{\alpha}_{1}+\bar{\alpha}_{2}+\bar{\alpha}_{3}+\bar{\alpha}_{4}
$$

where $\phi=(\sqrt{5}-1) / 2, \bar{\alpha}_{3}=9$ and $\bar{\alpha}_{4}=1$. We may recall that $\bar{\alpha}_{1}$ is the inverse electromagnetic coupling, $\bar{\alpha}_{2}$ is the inverse weak force coupling, $\bar{\alpha}_{3}$ is the inverse strong coupling and $\bar{\alpha}_{4}$ is the maximal quantum 
gravity coupling all measured at the electroweak scale and results in the exact theoretical $\bar{\alpha}_{o}=137.082039325$ inverse electromagnetic fine structure "constant". It is important to observe that $\bar{\alpha}_{i}$ seen as dimensions of a corresponding symmetry groups manifold add to exactly 100 because

$$
\sum_{i=1}^{4} \bar{\alpha}_{i}=60+30+9+1=100
$$

This could be interpreted as the normed total dimensions of the universe and could be sub-divided into the following [40]-[43]:

- $D_{1}=4 \%$ for spacetime of ordinary energy;

- $D_{2}=26 \%-4 \%=22 \%$ for compactified bosonic dimensions of dark matter energy;

- $D_{3}=100 \%-26 \%=74 \%$ for the pure dark energy.

Again this agrees with a very high accuracy with the COBE, WMAP and Type 1a supernova measurements and observations [130]-[133] and of course, also with Dvoretzky's theorem [39]-[41].

\section{The Self Similarity behind Newton's $E=1 / 2 m v^{2}$, Einstein's $E=m c^{2}$ and El Naschie's $E(O)=m c^{2} / 22, E(D)=m c^{2}(21 / 22)$ and $E(O)+E(D)=m c^{2}$}

A cow is definitely not a sphere but for all topological purposes it may be approximated to a sphere and glossing over biologically indispensible holes as well as the four stretched legs. This may be a drastic way to start arguing for an overwhelming self similarity of the cosmos and may also be the mathematical equations describing the cosmos. From this somewhat too general view point to be of any practical value, the manifest similarity between Newton's kinetic energy $E=1 / 2 m v^{2}$ on the one side and celebrated Einstein's maximal energy equation $E$ $=m c^{2}$ and El Naschie's two components equations $E(O)=m c^{2} / 22$ and $E(D)=m c^{2}(21 / 22)$ which sum up to that of Einstein $E(O)+E(D)=m c^{2}$ [36]-[43], on the other side it is difficult not to dream of finding a universal scaling law which explains that all celestial objects as small as a meteorite and as large as a galaxy may be regarded topologically as spheres with three spatial dimensions and two dimensional surface [36]-[43].

The first step in this direction could have been showing that four dimensions seems to be the expectation number of weighted integers from zero to infinity in the sense of E-infinity theory. This is really the reason behind the E-infinity Hausdorff dimension expectation [36]-[43] value

$$
\left\langle d_{i}\right\rangle=(1 / \phi)^{3}=4+\phi^{3}
$$

where $4+\phi^{3}$ is also the Hausdorff dimension of so that we may write [10]

$$
d_{c}^{(4)}=\left\langle d_{c}\right\rangle=4+\phi^{3}=4.23606 \simeq 4
$$

In such a space with infinite hierarchal topological dimensions, self similarity is basic and most fundamental so that it should not come as a surprise to observe that all formulas related to something as fundamental as energy $(E)$ should be subject to a minimum of self similarity or self affinity. To stress the point we recall that E-infinity spacetime is made of an infinite number of unions and intersection of random Cantor sets with a Hausdorff dimension equal to $\phi^{n}$ where $\mathrm{n}$ runs from zero to infinity. In turn Hausdorff dimensions are a measure of complexity and therefore are deeply related to entropy and consequently energy ergo the property of Cantorian self similarity is expected to be passed over to energy which means passed over to the equations defining energy. Could such a thought have passed in the mind of the great Albert Einstein? This might be idle or even far fetched unscientific speculation. However there is a curious and partially amusing story which could lead one to think that such ideas may really have crossed the great mind of Einstein (see Figure 4(a) and Figure 4(b). The said story is recorded in the classical and wholly enchanting book of the notable German American physicist Manfred Schroeder "Fractals, Chaos and Power Laws" [6]. In there Schroeder tells the story of how Einstein found a self similarity proof for the theorem of Pythagoras and shows that this part of Einstein's proof includes a formally identical formula to $E=m c^{2}$ (see Figure 6). However in all fairness to Schroeder he said that it is a mere coincidence and has nothing to do with the real $E=m c^{2}$ except the appearance and that $E=m c^{2}$ can be meaningful only in the context of Lorentzian invariance. On this count however we beg to differ. Admittedly biased by our own result $E=m c^{2} / 22+m c^{2}(21 / 22)$ [36]-[43] and our research on the subject we see here far more deep reasons than a coincidence as we will momentarily explain [36].

Let us start by quoting what is written on page 4 of the excellent book of Schroeder [6] 
"The 'resemblance' of equation 3 to Einstein's later discovery, his famous $E=m c^{2}$, is of course entirely fortuitous. The equivalence of mass $m$ and energy $E$ which is at the basis of nuclear power in all its guises is a consequence of Lorentz invariance. This invariance which underlies special relativity was predicted by Einstein in 1905 after it seems, several false starts.....".

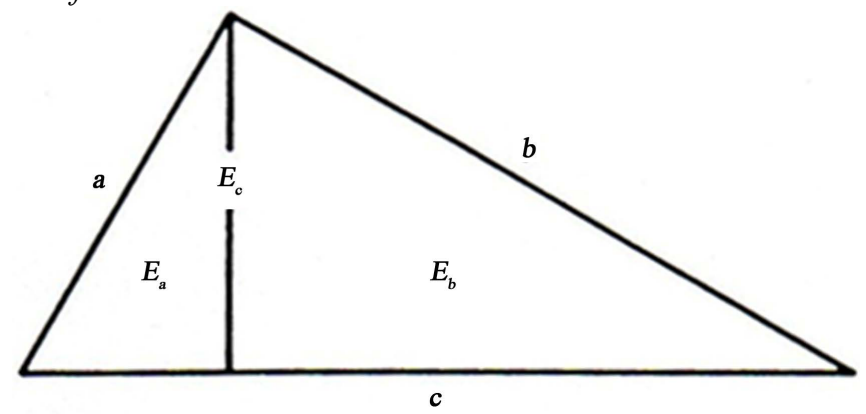

Figure 6. Pythagoras' theorem sketch for proof by the 11-year-old Einstein based on self similarity (see Section 8 of the present work as well as Ref. [6]).

Now analysing the above in a liberal way consistent with the big fuzzy picture approach of E-infinity, we can take the resemblance to mean self similarity or self affinity while the Lorentz invariance could be understood as a gauge which means scaling and renormalization and these notions are in turn just another more or less sophisticated form of self similarity which Einstein used to devise an alternative proof for the Pythagoras theorem [6]. In fact this theorem of Pythagoras is indispensible in giving a simple derivation of $E=m c^{2}$ as used in another excellent popular book on the subject by Brian Cox and Jeff Forshaw "Why Does $E=m c^{2}$ " [36]-[43]. With all of that in the back of our minds, looking at $E=1 / 2 m v^{2}$ of Newton compared to $E=m c^{2}$ of Einstein we do not need much persuasion to see that it is only a matter of scale where the $1 / 2 \rightarrow 1$ because we are integrating in the case of $v=c$ over a constant velocity, namely that of light and $v \rightarrow c$ is also a matter of scale. For this reason maximal energy equation $E=m c^{2}$ and El Naschie's two components equations $E(O)=(1 / 22) m c^{2}$ and $E(D)=$ $(21 / 22) m c^{2}$ could be seen as scaling of $E=m c^{2}$ when due to certain conditions the Lorentzian factor $\gamma=1$ of maximal "Einstein" energy takes two other values $\gamma(O)=1 / 22$ and $\gamma(D)=21 / 22$ for ordinary and dark energy respectively. Seen in this way it is quite conceivable, even though it is not very likely, that Einstein could have discovered the general form of E, namely [36]-[43] $E=\left(m c^{2} / 22\right)+m c^{2}(21 / 22)=m c^{2}$ long ago. After all it is sometimes good to think superficially. This dialectic statement could be illustrated by a well known observation of Einstein himself who explained time as that quantity that we measure with a clock. A second not so good example of the above is that when $v$ is constant then it goes out of the integration belonging to the definition of energy and $1 / 2$ becomes unity and $\mathrm{v}$ may be replaced by a new notation, namely the constant $\mathrm{c}$ so that at the end $E=1 / 2 m v^{2}$ is replaced by $E=m c^{2}$. From a formal view point that is really all and our conclusion is that self similarity and generalized equivalence relations [106] [108]-[111] are far more powerful scientific tools than we could have imagined before the advent of chaos, fractals and nonlinear dynamics.

To end this section we should add that according to Manfred Schroeder, the story of Einstein's proof of Pythagoras' theorem was recounted to him by SchneiovLifson of the Weizmann Institute in Tel Aviv who had it from Einstein's assistant Ernst Strauss who was told the story by Albert Einstein himself [6]. Similar to Newton discovering gravity by watching an apple falling from a tree, the Einstein story is so nice that it should be true even if it were not. Anyway right or wrong, this story is what almost compelled the present author to write the present paper partially and at a minimum as a tribute to the great Albert Einstein who always insisted that there are enough reasons, history, economics and space for Moslems, Christians and Jews to live together in the Holy Land, something which the present author finds marvellous and of course, possible.

\section{Self Similarity in Newton Gravity and Quantum Gravity}

It is remarkable that our self similarity principle which we used to justify moving from $E=1 / 2 m v^{2}$ to $E=$ $\left(m c^{2} / 22\right)+m c^{2}(21 / 22)=m c^{2}$ can be extended to the realm of Newton gravity and quantum gravity [36]-[43]. In the particular case considered here, quantum gravity inverse coupling constant can easily be seen following our E-infinity derivation to be the ratio between the total number of isometries of the E8E8 quantum spacetime and 
the total number of isometries at the energy scale of the standard model. This means [36]-[43]

$$
\bar{\alpha}_{Q g}=\frac{|E 8 E 8|}{|S U(3) S U(2) U(1)|} \simeq \frac{496}{12} \simeq 41.3
$$

which is very close to most of the results found in the literature in the non-super symmetric case. Similarly the number of isometries of the manifold combining classical gravity with all other fundamental forces is a staggering number, namely $10^{19}$ as we will show later on. We also know that at the point of total unification of all fundamental forces we are simply dealing with the Planck energy scale and that the coupling constant in this case is a maximum equal 1 . This is the only degree of freedom. Thus the equation corresponding to [47]

$$
\bar{\alpha}_{Q g}=\frac{496}{12}
$$

must now be [74]

$$
\bar{\alpha}_{\text {Planck }}=\frac{10^{19}}{\text { Newton degrees of freedom }}
$$

That means

$$
1 \simeq \frac{10^{19}}{\text { Newton degrees of freedom }}
$$

In other words Newton's degrees of freedom is $\simeq 10^{19}$. The next step is somewhat subtle and needs delicate consideration. We know that $S U(3) S U(2) U(1)=12$ is actually the square root of the inverse electromagnetic constant. The accurate transfinite expression is really [26]-[36]

$$
|S U(3) S U(2) U(1)|=\sqrt{\bar{\alpha}_{o}}=\sqrt{137.0820393}=11.7082039325 \simeq 12
$$

It follows then that

$$
\sqrt{\bar{\alpha}_{\text {Newton })}} \cong 10^{19}
$$

That means

$$
\bar{\alpha}_{\text {(Newton) }} \cong\left(10^{19}\right)^{2} \cong 10^{38}
$$

in a rather excellent agreement with the inverse value of the dimensionless Newtonian gravity. As for the spacetime manifold with $10^{19}$ isometries which we mentioned earlier on, this was investigated about eight years ago [26]-[36] and named Finkelstein-Clifford manifold. The main idea behind this very large number starts from looking at how many Planck length mini black holes $10^{-33} \mathrm{~cm}$ could fit into a proton wave length $10^{-14} \mathrm{~cm}$ which means $10^{-33} / 10^{-14}=10^{19}$ or alternatively how many proton masses $m \cong 939 \mathrm{MeV}$ fits into a Planck mass $10^{19} \mathrm{GeV}$ which means nearly $10^{19} \mathrm{GeV} / 1 \mathrm{GeV}=10^{19} \quad[26]-[36]$.

\section{A Cosmic Dirac's Vacuum as the Origin of Dark Energy and Dark Matter-A Speculative Scientific Idea}

Let us attempt to extend our present concepts and analysis to become truly cosmic. The idea or rather our hypothesis is that similar to the quantum mechanical Dirac's vacuum or "sea" which led to the prediction and subsequent discovery of the positron and thus to anti-particles, one could propose that the cosmos is abound with regions of positive energy and regions of negative energy. Since almost $95.5 \%$ of the energy density is due to the quantum wave, i.e. quantum energy of propagation and since the quantum wave is the cobordism of the quantum particle, then it follows that most of the energy will be located at the boundary of the holographic boundary of the universe that lies at the hyperbolic infinity [52] [53]. The same conclusion may also be reached by invoking Dvoretzky's theorem of measure concentration. Our first basic assumption is one adopted by almost all theories dealing with modern quantum cosmology, namely that our entire universe is described by the E8E8 exceptional 
Lie symmetry group and its associated fuzzy manifold with $496-k^{2}$ Hausdorff dimension where $k=\phi^{3}\left(1-\phi^{3}\right)$ is 'tHooft's renormalon [29] [59]. We note further that $k=2 \phi^{5}$ where $\phi^{5}$ is Hardy's probability of the quantum entanglement of two particles and $\phi=(\sqrt{5}-1) / 2$ is the Hausdorff dimension of the zero set quantum particle. Second we must recall the formula for the degrees of freedom of pure gravity, i.e. the formula of the Vierbien description of graviton, namely [59] [116]

$$
D(\text { vacuum })=d(d-3) / 2
$$

It is then a trivial matter to reason that for tangible energy and matter $d$ must be $d=4$ which leads to $D=2$ while the full space of Witten's M-theory may be found from $d=11$ to be

$$
D=11(11-3) / 2=44 \text {. }
$$

Consequently the density Lorentz factor must be

$$
\gamma_{(o)}=\frac{D=2}{D=44}=\frac{1}{22}
$$

for ordinary matter and energy and

$$
\gamma_{(D)}=1-\frac{1}{22}=\frac{21}{22}
$$

for dark matter and energy as reasoned in previous sections. Here however we want to expand our argument and start with something more insightful than $E=m c^{2}$, namely the formula from which Einstein deduced $E=m c^{2}$, namely [46]

$$
E^{2}=\left(m c^{2}\right)^{2}+A
$$

where $A=p^{2} c^{2}$ and $P=m v$ is the momentum [46]. For $A=0$ we retrieve our original formula put with the vital or subtle plus or minus because of the trivial mathematics with nontrivial physical consequences, namely taking the root of $\mathrm{E}^{2}$ and finding that

$$
E=\sqrt{\left(m c^{2}\right)^{2}}= \pm m c^{2}
$$

That way we can relate the previous discussion and the finding $E= \pm m c^{2}$ to the double Lie symmetry group E8E8 where the first E8 will be assigned to our universe while the second one will be assigned to the so called shadow universe [112] where we are arguing that the "missing" dark energy resides. From the preceding analysis we can thus assume with considerable confidence that when using $E^{2}$ then we must note that while our universe is fully described by E8E8 we should subtract from it either $D=4$ of Einstein spacetime in an exact analysis or alternatively we take out the 12 messenger particles of the standard model in an integer approximate analysis. Similarly we note for the same purpose that for the dark section we have to subtract the 44 degrees of freedom of pure gravity plus the 11 dimension of M-theory, i.e. we will be left with $496-(44+11)=441$ de grees of freedom or isometries. Combining what we have just calculated with $E^{2}$ one finds the squared dark energy density of the cosmos as

$$
E^{2}(D)=\frac{|E 8 E 8|-55}{|E 8 E 8|-12} m^{2} c^{4}=\frac{441}{484} m^{2} c^{4}
$$

It is then rather gratifying to find our previous result strongly confirmed by

$$
E(D)=\sqrt{\frac{441}{484}} m c^{2}=\frac{\sqrt{441}}{\sqrt{484}} m c^{2}= \pm 21 / 22 m c^{2}
$$

exactly as expected but with an additional negative sign besides the well known positive sign. On the other hand Einstein's celebrated formula follows as a trivial result when we ignore the true vacuum, i.e. pure gravity as well as the $|S U(3) S U(2) U(1)|=12$ of the standard model and find 


$$
E(\text { Einstein })=\frac{ \pm|E 8 E 8|}{|E 8 E 8|} m c^{2}= \pm m c^{2}
$$

So far for the integer theory but the really very nice aspect of our theory comes to the fore when we considered the exact transfinite analysis and discovered the role of dimensional regularization of that at the time young Dutch Ph.D. student and presently senior Nobel laureate Gerardus ‘tHooft [121]-[123].

\section{The Amazing Role of 'tHooft's Renormalon $k=\phi^{3}\left(1-\phi^{3}\right)$ and the \\ Exact Transfinite Analysis in an Essentially Fractal Spacetime [117]}

In the preceding analysis we used for dim E8E8 the integer value 496 and invoked it in finding $E^{2}$. However the exact transfinite value is not 496 but $496-k^{2}$, where $k=\phi^{3}\left(1-\phi^{3}\right)$. Proceeding in a transfinitely consistent way using the exact transfinite expression it is not difficult to show that $E(D)$ may be found exactly from

$$
E(D)=\sqrt{\frac{\left(496-k^{2}\right)-[55-(2)(4-k)]}{\left(496-k^{2}\right)-4}}
$$

where $4-k$ is the dimensionality of 'tHooft-Veltman-Wilson fractal renormalization spacetime and $k=\phi^{3}\left(1-\phi^{3}\right)=0.18033989$ is the charge of 'tHooft's renormalon [29] [117]. That way we find

$$
E(D)=\sqrt{\frac{448.6067977}{491.967775}}= \pm \frac{21+k}{22+k}
$$

Now it comes as no surprise to note that we could have cut the preceding analysis to exactly two lines of trivial calculation by simply pausing a minute to reason that the energy density must be simply the ratio of a completely solid spacetime, i.e. Einstein's spacetime dimension $D=4$ to 'tHooft-Veltman-Wilson fractal spacetime $D=4-k$ which has uncountably infinitely many gaps like a Menger spongeso that the dark energy density is given simply by

$$
E(D)=\frac{4-k}{4} m c^{2}=\frac{(10)\left(\phi^{2}\right)}{(2)^{2}} m c^{2}=(5)(\phi)^{2} / 2 m c^{2}=\frac{21+k}{22+k} m c^{2}
$$

The drawback of this shortcut is that the plus minus double sign of taking the square root of E is not as obvious as in the previous analysis.

\section{Non-Standard Riemannian Stein Spaces for Ordinary and Dark Energy Density}

It is useful to reformulate our preceding analysis in terms of combinatorics. This way we can write [46] [124]

$$
\left(\begin{array}{c}
11 \\
1
\end{array}\right)+\left(\begin{array}{c}
11 \\
2
\end{array}\right)+\left(\begin{array}{c}
11 \\
5
\end{array}\right)=528
$$

which is the total quantum-like state of Witten's 5-Brane in eleven dimensional theory which is also given by [46]

$$
N_{11}^{(32)}=(32)(33) / 2=528
$$

killing vector fields. Furthermore we have

$$
\left(\begin{array}{c}
11 \\
0
\end{array}\right)+\left(\begin{array}{c}
11 \\
3
\end{array}\right)+\left(\begin{array}{c}
11 \\
4
\end{array}\right)=496
$$

which is equal to the dimension of E8E8 as well as $\mathrm{SO}(32)$ where [124]

$$
|S O(32)|=(32)(31) / 2=496
$$


Adding the two results together one finds that

$$
|E 8 E 8|+N_{11}^{(32)}=496+528=1024
$$

which is nothing but a membrane in eleven dimensions which gives us the remarkable E-infinity result

$$
d_{c}^{(n)}=\left(1 / d_{c}^{(o)}\right)^{n-1}
$$

For $n=11$ and $d_{c}^{(o)}=1 / 2$ this leads to

$$
d_{c}^{(11)}=(2)^{10}=1024
$$

That way we find a simple combinatoric Brane formula for dark energy via

$$
[E(D)]^{2}=\frac{\left(\begin{array}{c}
11 \\
0
\end{array}\right)+\left(\begin{array}{c}
11 \\
3
\end{array}\right)+\left(\begin{array}{c}
11 \\
4
\end{array}\right)-\left(\begin{array}{c}
11 \\
2
\end{array}\right)}{\left(\begin{array}{c}
11 \\
0
\end{array}\right)+\left(\begin{array}{c}
11 \\
3
\end{array}\right)+\left(\begin{array}{c}
11 \\
4
\end{array}\right)-\left[\left(\begin{array}{c}
11 \\
0
\end{array}\right)-\left(\begin{array}{c}
11 \\
1
\end{array}\right)\right]}=\frac{496-55}{496-12}=\frac{441}{484}
$$

This means

$$
E(D)= \pm \sqrt{\frac{441}{484}}= \pm 21 / 22
$$

exactly as it should be. The corresponding exact transfinite expression is

$$
E(D)=\frac{4-k}{4}=1-k / 4
$$

and since $k=2 \phi^{5}$ then

$$
E(D)=1-\phi^{5} / 2
$$

where $\phi^{5}$ is Hardy's quantum entanglement of two particles while $\phi^{5} / 2$ is the topological characteristic value of the entangleon corresponding to $\mathrm{k}$ of 'tHooft's renormalon hypothetical particle of dimensional regularization. Finally, motivated by the facts that

$$
|E 8|+|E 7|+|E 6|+(|E 5|=|S O(10)|=45)+(|E 4|=|S O(5)|=24)=528
$$

and that our string bosonic spacetime is $\mathrm{D}=26$ similar to one of the two directions of Heterotic superstrings, we will use a two Stein space representing a non-standard Riemannian manifold with a Lie symmetry group dimension given by [119] [135]

$$
\operatorname{dim}(E 6(-26)=E 6 / F 4)=26
$$

where $|E 6|=133$ and $|F 4|=52$ correspond to the well known exceptional Lie groups family. Noting further more that for the non-compactified Klein-Kaluza section of $D=5$ and the Einstein section $D=4$ we have first the two Stein spaces, namely first:

$$
\operatorname{dim}\left(A_{2}^{R}=\frac{S U(3)}{S O(3)}\right)=5
$$

and second the simply connected symmetric harmonic $\mathrm{k}=1$ Stein space, i.e. Einstein space [119]

$$
\operatorname{dim}\left[S^{4}=\frac{S O(4+1)}{S O(4)}\right]=4
$$

then we can write the ordinary and the dark energy Lorentz factor $\gamma(o)$ and $\gamma(D)$ respectively as follows: 


$$
\gamma(o)=\frac{1|U(1)|}{26-4}=\frac{1}{22}
$$

and

$$
\gamma(D)=\frac{26-5}{26-4}=\frac{21}{22}
$$

where $\gamma(o)+\gamma(D)=\gamma($ Einstein $)=1$ leading to $E=m c^{2}$. Some readers may find the following relation interesting in their own right within the context of dark energy research

$$
|F 4|=52,|F 4(+4)|=24
$$

Thus

$$
\begin{aligned}
& |F 4(+4)|-|S U(2)|=24-3=21 \\
& |F 4|+|S U(2)|=52+3=55
\end{aligned}
$$

and finally

$$
\left|G_{2}\right|+|S U(3)|=14+8=22
$$

In addition we draw the readers' attention to identical results obtained by the author several years ago using the holonomy and co-holonomy of Kähler manifolds where the ratio of the relevant Betti number for smooth Einstein manifold is one and for a rugged Kähler is 22 leading to $\gamma(o)=1 / 22$ [35].

\section{Conclusion without a Final Word}

Although the great man spoke German with a characteristic Danish accent, the author could not translate the eloquence of Niels Bohr's German sentence as recounted in the memories of the young Werner Heisenberg [113]:

"Nur die FüllefürtzurKlarheit".

In English this could mean something like "only abundance leads to clarity". The somewhat contradictory statement reflects of course Niels Bohr's admiration for F. Hegel's dialectic philosophy [113]. However it also reflects the essence of the present paper and the philosophy of "simplexity of complexity" proposed here. Summarizing our present contribution we may say the following.

Starting from nonlinear dynamics, chaos and fractals and linking that with the pure mathematical results of the theory of equivalence relations [108]-[111], the present work takes very seriously several ideas revolving around self similarity and scaling. Within such theories we regard nature as generically fuzzy and almost pathological at the fundamental level [7] [65]. We take then the view that these ideas are by no means entirely new but were thought about deeply probably by Albert Einstein and for sure by the great Russian-American academician G.I. Barenblatt who wrote in his book entitled "Simply Scaling" [106] "every mathematical model is based on "intermediate asymptotic". Adding this to what the French Fields Medalist and exceptional mathematician and theoretical physicist A. Connes [107] wrote about the pathological Penrose universe [46] [65] the main thrust of the present paper becomes clearer. Continuing our scientific journey from this location we realize that the pathway taking us from Newton's kinetic energy to the new Einstein ordinary energy and dark energy density of the universe is exceedingly shorter than the long way to Tipperary. It maybe that it was the wonderful company of the many references cited herein which made the journey appear to the author to be shorter than it was [1]-[135]. Incidentally just as the author finished writing this paper that the good news about finding gravitational waves was announced [43]. This is of course not a proof that Einstein's general theory of relativity is correct because we know from many other experiments that it is correct. However the finding is a very strong proof that spacetime is physically real just as the Casimir effect is so our spacetime energy reactor now stands on even firmer ground [136].

\section{Acknowledgements}


The author is deeply indebted to the work of Prof. G. 'tHooft on dimensional regularization which is far more than an ingeniously simple mathematical manoeuvre concealing a great deal of physical reality about the fine structure of quantum spacetime. He is also grateful to all the discoverers of the completely beautiful theory of strong interaction that has clear marks on the present paper. Last but not least, the work of A. Connes was indispensible for the present author.

\section{References}

[1] B. Mandelbrot: The fractal geometry of nature. Freeman, New York, USA, 1977.

[2] P.S. Addison: Fractals and chaos: An illustrated course. IOP, Bristol, UK, 1997.

[3] H.O. Peitgen, H. Jürgens and D. Saupe: Chaos and fractals. Springer, New York, USA, 1992.

[4] J. Guckenheimer and P. Holmes: Nonlinear oscillations, dynamical systems and bifurcations of vector fields. Springer, New York, USA, 1983.

[5] M.C. Gutzwiller: Chaos in classical and quantum mechanics. Springer, New York, USA, 1990.

[6] M. Schroeder: Number theory in science and communication: With applications in cryptographys, physics, digital information, computing and self similarity. Springer Verlag, Berlin, Germany, 2009.

[7] L. Marek-Crnjac, M.S. El Naschie and J.-H. He: Chaotic fractals at the root of relativistic quantum physics and cosmology. International Journal of Modern Nonlinear Theory and Application, 2, 2013, pp. 78-88.

[8] M.S. El Naschie: The concepts of E-infinity: An elementary introduction to the Cantorian-fractal theory of quantum physics. Chaos, Solitons \& Fractals, 22, 2004, pp. 495-511.

[9] M.S. El Naschie: Silver mean Hausdorff dimension and Cantor sets. Chaos, Solitons \& Fractals, 4(10), 1994, pp. 1861-1869.

[10] M.S. El Naschie: A review of E-infinity theory and the mass spectrum of high energy particle physics. Chaos, Solitons \& Fractals, 19(1), 2004, pp. 209-236.

[11] M.S. El Naschie: Fuzzy dodecahedron topology and E-infinity spacetime as a model for quantum physics. Chaos, Solitons \& Fractals, 30(5), 2006, pp. 1025-1033.

[12] G. Iovane: Wave guiding and mirroring effects in stochastic self-similar ad Cantorian E-infinity Universe. Chaos, Solitons \& Fractals, 23(3), 2005, pp. 691-700.

[13] M.S. El Naschie: A guide to the mathematics of E-infinity Cantorian spacetime theory. Chaos, Solitons \& Fractals, 25(5), 2005, pp. 955-964.

[14] G. Iovane: Self-similar and oscillating solutions of Einstein's equation and other relevant consequences of a stochastic self-similar and fractal universe via El Naschie's E-infinity spacetime. Chaos, Solitons \& Fractals, 23(2), 2005, pp. 351-360.

[15] Y. Tanaka, Y. Mizuno and T. Kado: Chaotic dynamics in the Friedmann equation. Chaos, Solitons \& Fractals, 24(2), 2005, pp. 407-422.

[16] M.S. El Naschie: Nuclear spacetime theories, superstrings, monster group and applications, Chaos, Solitons \& Fractals, 10(2-3), 1999, pp. 567-580.

[17] M.S. El Naschie: Gravitational instanton in Hilbert space and the mass of high energy elementary particles. Chaos, Solitons \& Fractals, 20(5), 2004, pp. 917-923.

[18] P. Cilliers and D. Spurrett: Complexity and post-modernism: Understanding complex systems. South African Journal of Philosophy, 18(2), 1999, pp. 258-274.

[19] E. Bertschinger: Self-similar secondary infall and accretion in an Einstein-de Sitter universe. The Astrophysical Journal Supplement Series, 58, 1985, pp. 39-66.

[20] E. Bertschinger: Self-similar evolution of hole in an Einstein-de Sitter universe. The Astrophysical Journal Supplement Series, 58, 1985, pp. 1-37.

[21] C.R. Evans and J.S. Coleman: Critical phenomena and self-similarity in the gravitational collapse of radiation fluid. Physical Review Letters, 72, 1994, pp. 1782-1785.

[22] A. Ori and T. Piran: Naked singularities and other features of self-similar general-relativistic gravitational collapse. Physical Review D, 47, 1990, pp. 777-780.

[23] A.J. Einstein, H.S. Wu and J. Gil: Self-affinity and lacunarity of chromatin texture in benign and malignant breast epithelial cell nuclei. Physical Review Letters, 80, 1998, pp. 397-400.

[24] B.J. Carr and A.A. Coley: Self-similarity in general relativity. Classical and Quantum Gravity, 16(7), 1999, pp. 
R31-R71.

[25] J.A. Fillmore and P. Goldreich: Self-similar gravitational collapse in an expanding universe. The Astrophysical Journal, 281, 1984, pp. 1-8.

[26] M.S. El Naschie: On 't Hooft dimensional regularization in E-infinity space. Chaos, Solitons \& Fractals, 12(5), 2001, pp. 851-858.

[27] M.S. El Naschie: The VAK of vacuum fluctuation, spontaneous self organization and complexity theory interpretation of high energy particle physics and the mass spectrum. Chaos, Solitons \& Fractals, 18(2), 2003, pp. 579-605.

[28] M.S. El Naschie: Elementary prerequisites for E-infinity (Recommended background readings in nonlinear dynamics, geometry and topology). Chaos, Solitons \& Fractals, 30, 2006, pp. 579-605.

[29] M.S. El Naschie: On a new elementary particle from the disintegration of the Symplectic 't Hooft-Veltman-Wilson fractal spacetime. World Journal of Nuclear Science and Technology, 4(4), 2014, pp. 216-221.

[30] G. Iovane: El Naschie E-infinity Cantorian spacetime and length scales in cosmology. International Journal of Nonlinear Sciences and Numerical Similation, 7(2), 2006, pp. 155-162.

[31] L. Marek-Crnjac, J.-H. He: An invitation to El Naschie's theory of cantorian space-time and dark energy. International Journal of Astronomy and Astrophysics, 3(4), 2013, pp. 464-471.

[32] A.R. El-Nabulsi: Fractional action-like variational problems in holonomic, non-holonomic and semi-holonomic constrained and dissipative dynamical systems. Chaos, Solitons \& Fractals, 42(1), 2009, pp. 52-61.

[33] A.R. El-Nabulsi: Fractional dynamics, fractional weak bosons masses and physics beyond the standard model. Chaos, Solitons \& Fractals, 41(5), 2009, pp. 2262-2270.

[34] M.S. El Naschie: The measure concentration of convex geometry in a quasi Banach spacetime behind the supposedly missing dark energy of the cosmos. American Journal of Astronomy \& Astrophysics, 2(6), 2014, pp. 72-77.

[35] M.S. El Naschie: A unified Newtonian-relativistic quantum resolution of supposedly missing dark energy of the cosmos and the constancy of the speed of light. International Journal of Modern Nonlinear Theory \& Application, 2, 2013, pp. 43-54.

[36] A.J. Babchin and M.S. El Naschie: On the real Einstein beauty $\mathrm{E}=\mathrm{kmc}^{2}$. World Journal of Condensed Matter Physics, 6(1), 2016, pp. 1-6.

[37] L. Marek-Crnjac: On El Naschie's fractal-cantorian space-time and dark energy-A tutorial review. Natural Science, 7(13), 2015, pp. 581-598.

[38] M.S. El Naschie: Casimir-dark energy nano reactor design proposal based on fractals. International Journal of Innovation is Science and Mathematics, 3(4), 2015, pp. 187-194.

[39] M.S. El Naschie: The Counterintuitive increase of information due to extra spacetime dimensions of a black hole and Dvoretzky's theorem. Natural Science, 7(10), 2015, pp. 483-487.

[40] M.S. El Naschie: Application of Dvoretzky's theorem of measure concentration in physics and cosmology. Open Journal of Microphysics, 5, 2015, pp. 11-15.

[41] M.S. El Naschie: A resolution of the black hole information paradox via transfinite set theory. World Journal of Condensed Matter Physics, 5, 2015, pp. 249-260.

[42] M.S. El Naschie: If quantum "wave" of the universe then quantum "particle" of the universe: A resolution of the dark energy question and the black hole information paradox. International Journal of Astronomy \& Astrophysics, 5, 2015, pp. 243-247.

[43] M.S. El Naschie: Quantum fractals and the Casimir-dark energy duality-The road to a clean quantum energy nano reactor. Journal of Modern Physics, 6, 2015, pp. 1321-1333.

[44] M.S. El Naschie: From fusion algebra to cold fusion or from pure reason to pragmatism. Open Journal of Philosophy, 5(6), 2015, pp. 319-326.

[45] M.S. El Naschie: The Casimir effect as a pure topological phenomenon and the possibility of a Casimir nano reactor-A preliminary design. American Journal of Nano Research and Application, 3(3), 2015, pp. 33-40.

[46] R. Penrose: The road to reality. J. Cape, London, UK, 2004

[47] S. Hawking and W. Israel: 300 years of gravitation. Cambridge University Press, Cambridge, UK, 1990.

[48] S. Weinberg: Cosmology. Oxford University Press, Oxford, UK, 2008.

[49] M. Helal, L. Marek-Crnjac and J.-H. He: The three page guide to the most important results of M.S. El Naschie's research in E-infinity quantum physics. Open Journal of Microphysics, 3, 2013, pp. 141-145.

[50] M.S. El Naschie: Why E is not equal mc². Journal of Modern Physics, 5(9), 2014, pp. 743-750.

[51] M.S. El Naschie: Experimentally based theoretical arguments that Unruh's temperature, Hawking's vacuum fluctuation 
and Rindler's wedge are physically real. American Journal of Modern Physics, 2(6), 2013, pp. 357-361.

[52] M.S. El Naschie: The hyperbolic extension of Sigalotti-Hendi-Sharifzadeh's golden triangle of special theory of relativity and the nature of dark energy. Journal of Modern Physics, 4(3), 2013, pp. 354-356.

[53] M.S. El Naschie: A Rindler-KAM spacetime geometry and scaling the planck scale solves quantum relativity and explains dark energy. International Journal of Astronomy and Astrophysics, 3(4), 2013, pp. 483-493.

[54] M.S. El Naschie: Quantum entanglement: Where dark energy and negative, accelerated expansion of the universe comes from. Journal of Quantum Information Science, 3(2), 2013, pp 55-57.

[55] M.S. El Naschie: Feigenbaum scenario for turbulence and Cantorian E-infinity theory of high energy particle physics. Chaos, Solitons \& Fractals, 32(3), 2007, pp. 911-915.

[56] M.S. El Naschie: Elementary number theory in superstring loop quantum mechanics, twistors and E-infinity high energy physics. Chaos, Solitons \& Fractals, 27(2), 2006, pp. 297-330.

[57] M.S. El Naschie: A review of application and results of E-infinity. International Journal of Nonlinear Science \& Numerical Simulation, 2007, 8(1), pp. 11-20.

[58] M.S. El Naschie and A. Helal: Dark energy explained via the Hawking-Hartle quantum wave and the topology of cosmic crystallography. International Journal of Astronomy and Astrophysics, 3(3), 2013, pp. 318-343.

[59] M.S. El Naschie: Cosmic dark energy from 'thooft's dimensional regularization and Witten's topological quantum field pure gravity. Journal of Quantum Information Science, 4(2), 2014, pp. 83-91.

[60] E.J. Copeland, M. Sami and S. Tsujikawa: Dynamics of dark energy. International Journal of Modern Physics D, 15(11), 2006, pp. 1753-1935.

[61] M.S. El Naschie: Einstein-Rosen Bridge (ER), Einstein-Podolsky-Rosen Experiment (EPR) and Zero Measure Rindler-KAM Cantorian Spacetime Geometry (ZMG) are conceptually equivalent. International Journal of Quantum Information Science, 6(1), 2016, pp. 1-9.

[62] M.S. El Naschie: From $E=m c 2$ to $E=m c 2 / 22-A$ short account of the most famous equation in physics and its hidden quantum entangled origin. Journal of Quantum Information Science, 4, 2014, pp. 284-291.

[63] M.S. El Naschie: What is the missing dark energy in a nutshell and the Hawking-Hartle quantum wave collapse. International Journal of Astronomy and Astrophysics, 3(3), 2013, pp. 205-211.

[64] M.S. El Naschie: Superstrings, knots and noncommutative geometry in E-infinity space. International Journal of Theoretical Physics, 37(12), 1998, pp. 2935-2951.

[65] M.S. El Naschie: Penrose universe and Cantorian spacetime as a model for noncommutative quantum geometry. Chaos, Solitons \& Fractals, 1998, 9(6), pp. 931-933.

[66] M.S. El Naschie: Topics in the mathematical physics of E-infinity theory. Chaos, Solitons \& Fractals, 30(3), 2006, pp. 656-663.

[67] M.S. El Naschie: Advanced prerequisites for E-infinity theory. Chaos, Solitons \& Fractals, 30(12), 2006, pp. 636-641.

[68] M.S. El Naschie: Intermediate prerequisites for E-infinity theory. Chaos, Solitons \& Fractals, 30, 2006, pp. $622-628$.

[69] J.-H. He: Application of E-infinity theory to biology. Chaos, Solitons \& Fractals, 28(2), 2006, pp. 285-289.

[70] M.S. El Naschie: On the unification of heterotic strings, M theory and E ( $\infty$ ) theory. Chaos, Solitons \& Fractals, 11(14), 2000, pp. 2397-2408.

[71] M.S. El Naschie: On a class of general theories for higher energy particle physics. Chaos, Solitons \& Fractals, 2002, 14, pp. 649-668.

[72] M.S. El Naschie: Wild topology, hyperbolic geometry and fusion algebra of high energy particle physics. Chaos, Solitons \& Fractals, 13(9), 2009, pp. 1935-1945.

[73] M.S. El Naschie: The golden mean in quantum geometry, knot theory and related topics. Chaos, Solitons \& Fractals, 10(8), 1999, pp. 1303-1307.

[74] M.S. El Naschie: Quantum entanglement as a consequence of a Cantorian micro spacetime geometry. Journal of Quantum Information Science, 1, 2011, pp. 50-53.

[75] M.S. El Naschie: Von Neumann geometry and E-infinity quantum spacetime. Chaos, Solitons \& Fractals, 9(12), 1998, pp. 2023-2030.

[76] A. Sangalli: The importance of being fuzzy. Princeton University Press, Princeton, USA, 1998.

[77] S. Nanda and N.R. Das: Fuzzy mathematical concepts. Alpha Science, Oxford, UK, 2010.

[78] A.B. Balachandran, S. Kürkcüoglu and S. Vaidya: Lectures on fuzzy and fuzzy susy physics. World Scientific, Singapore, 2007. 
[79] N. Palanippan: Fuzzy topology. Alpha Science, Pangbourne, UK, 2002.

[80] M.S. El Naschie: Fuzzy knot theory interpretation of Yang-Mills instantons and Witten's 5-Brane model. Chaos, Solitons \& Fractals, 38(5), 2008, pp. 1349-1354.

[81] M.S. El Naschie: Quantum gravity, Clifford algebras, fuzzy set theory and the fundamental constants of nature. Chaos, Solitons \& Fractals, 20(3), 2004, pp. 437-450.

M.S. El Naschie: The discrete charm of certain eleven dimensional space-time theory. International Journal of Nonlinear Sciences and Numerical Simulation, 7(4), 2006, pp. 407-409.

[82] M.S. El Naschie: Fuzzy multi-instanton knots in the fabric of spacetime and Dirac's vacuum fluctuation. Chaos, Solitons \& Fractals, 38, 2008, pp. 1260-1268.

[83] M.S. El Naschie, S. Olsen, J.-H. He, S. Nada, L. Marek-Crnjac and A. Helal: On the need for fractal logic in high energy quantum physics. International Journal of Modern Nonlinear Theory and Application, 21(3), 2012, pp. 84-92.

[84] M.S. El Naschie: On the uncertainty of Cantorian geometry and the two-slit experiment. Chaos, Solitons \& Fractals, 19(3), 1998, pp. 517-529.

[85] M.S. El Naschie: On a fuzzy Kähler-like manifold which is consistent with the two slit experiment. International Journal of Nonlinear Sciences and Numerical Simulation, 6(2), 2005, pp. 95-98.

[86] M.S. El Naschie: Transfinite harmonization by taking the dissonance out of the quantum field symphony. Chaos, Solitons \& Fractals, 36, 2008, pp. 781-786.

[87] M.S. El Naschie and L. Marek-Crnjac: Deriving the exact percentage of dark energy using a transfinite version of Nottale's scale relativity. International Journal of Modern Nonlinear Theory and Application, 1, 2012, pp. 118-124.

[88] M.S. El Naschie: Towards a quantum golden field theory. International Journal of Nonlinear Sciences and Numerical Simulation, 8(4), 2007, pp. 477-482.

[89] M.S. El Naschie: Asymptotic freedom and unification in a golden quantum field theory. Chaos, Solitons \& Fractals, 36(3), 2008, pp. 521-525.

[90] M.S. El Naschie: An outline for a quantum golden field theory. Chaos, Solitons \& Fractals, 37(2), 2008, pp. $317-323$.

[91] M.S. El Naschie: Extended renormalizations group analysis for quantum gravity and Newton's gravitational constant. Chaos, Solitons \& Fractals, 35, 2008, pp. 425-431.

[92] J.-H. He and L. Marek-Crnjac: The quintessence of El Naschie's theory of fractal relativity and dark energy. Fractal Spacetime and Noncommutative Geometry in Quantum \& High Energy Physics, 3(2), 2013, pp. 130-137.

[93] M.S. El Naschie: Quantum golden field theory—-Ten theorems and various conjectures. Chaos, Solitons \& Fractals, 36, 2008, pp. 1121-1125.

[94] M.S. El Naschie: Transfinite electrical networks, spinoral varieties and gravity Q bits. International Journal of Nonlinear Sciences and Numerical Simulation, 5(3), 2004, pp. 191-198.

[95] M.S. El Naschie: Quantum gravity from descriptive set theory. Chaos, Solitons \& Fractals, 19(5), 2004, pp. $1339-1344$.

[96] M.S. El Naschie: On a transfinite symmetry group with 10 to the power of 19 dimensions. Chaos, Solitons \& Fractals, 36(3), 2008, pp. 539-541.

[97] M.S. El Naschie: On dualities between Nordstrom-Kaluza-Klein, Newtonian and quantum gravity. Chaos, Solitons \& Fractals, 36(4), 2008, pp. 808-810.

[98] M.S. El Naschie: The quantum gravity Immirzi parameter-A general physical and topological interpretation. Gravitation and Cosmology, 19(3), 2013, pp. 151-155.

[99] M.S. El Naschie: High energy physics and the standard model from the exceptional Lie groups. Chaos, Solitons \& Fractals, 36(1), 2008, pp. 1-17.

[100] C. Castro: Quantum space-time infinite dimensional. Chaos, Solitons \& Fractals, 11(11), 2000, pp. 1663-1670.

[101] M.S. El Naschie: The exceptional Lie symmetry groups hierarchy and the expected number of Higgs bosons. Chaos, Solitons \& Fractals, 35(2), 2008, pp. 268-273.

[102] M.S. El Naschie: Exceptional Lie groups hierarchy and the structure of the micro universe. International Journal of Nonlinear Sciences and Numerical Simulation, 8(3), 2007, pp. 445-450.

[103] M.S. El Naschie: Notes on exceptional Lie groups hierarchy and possible implications for E-infinity. Chaos, Solitons \& Fractals, 35(1), 2008, pp. 67-70.

[104] M.S. El Naschie: Symmetry group prerequisite for E-infinity in high energy physics. Chaos, Solitons \& Fractals, 35(1), 2008, pp. 202-211.

[105] M.S. El Naschie: Quasi exceptional E12 Lie symmetry group with 685 dimensions, KAC-Moody algebra and E-infinity Cantorian spacetime. Chaos, Solitons \& Fractals, 38(4), 2008, pp. 990-992. 
[106] G.I. Barrenblatt: Scaling. Cambridge University Press, Cambridge, UK, 2003.

[107] A. Connes: Noncommutative geometry. Academic Press, San Diego, USA, 1994 (see in particular pages 88-93).

[108] M. Nakahara: Geometry, topology and physics. Second Edition, Tayler and Francis, New York, USA, 2003.

[109] J. Sampo and P. Luukka: Similarity classifier with generalized mean: Ideal vector approach in "Fuzzy Systems and Knowledge Discovery". Series Lecture Notes in Computer Sciences, 4423, 2006, pp. 1140-1147.

[110] P. Lukka: Similarity classifier using similarities based on modified probabilistic equivalence relation. Knowledge Based Systems, 22(1), 2009, pp. 57-62.

[111] S.K. Parhi: Modification of generalization of equivalence relation. IOSR Journal of Mathematics, 9(1), 2013, pp. 70-73.

[112] F.D. Peat: Superstrings. Abacus Books, London, UK, 1988.

[113] W. Heisenberg: Der Teil und das Ganze. Piper-Verlag, Munich, Germany, 1969.

[114] M.S. El Naschie: Stress, stability and chaos in structural engineering: An energy approach. McGraw Hill, London, UK, 1990.

[115] M.S. El Naschie: Mathematical models and methods in dark energy theory: Dvoretzky's theorem, Casimir effect, Mobius geometry. Problems of Nonlinear Analysis in Engineering Systems, 2(44), 2015, pp. 1-16. University of Kazan Press, Russia. (Published in Russian and English language versions).

[116] M.S. El Naschie: Asymptotically safe pure gravity as the source of dark energy of the vacuum. International Journal of Astrophysics and Space Science, 2(1), 2014, pp. 12-15.

[117] M.S. El Naschie: Compactified dimensions as produced by quantum entanglement, the four dimensionality of Einstein's smooth spacetime and 'tHooft's 4- $\varepsilon$ fractal spacetime. American Journal of Astronomy and Astrophysics, 2(3), 2014, pp. 34-37.

[118] M.S. El Naschie: The self referential pointless universe geometry as the key to the resolution of the black hole information paradox. International Journal of Innovation in Science \& Mathematics, 3(5), 2016, pp. 254-256.

[119] T. Willmore: Riemannian geometry. Oxford University Press, Oxford, UK, 1993.

[120] R. Gilmore: Lie groups, physics and geometry. Cambridge University Press, Cambridge, UK, 2008.

[121] G. 'tHooft: In search of the ultimate building blocks. Cambridge University Press, Cambridge, UK, 1997.

[122] G. 'tHooft: 50 years of Yang-Mills theory. World Scientific, Singapore, 2005.

[123] G. 'tHooft: Under the spell of gauge principle. World Scientific, Singapore, 1994.

[124] M. Duff (Editor): The world in eleven dimensions. IOP Publishing, Bristol, UK, 1999.

[125] D. Freedman and A. van Proeyen: Super gravity. Cambridge University Press, Cambridge, UK, 2004.

[126] P. West: Introduction to strings and branes. Cambridge University Press, Cambridge, UK, 2012.

[127] L. Smolin: Three roads to quantum gravity. Weindenfeld \& Nicolson, London, UK, 2000.

[128] J. Magueijo: Faster than the speed of light. William Heinemann, London, UK, 2003.

[129] L. Susskind: The black hole war. Back Bay Books, New York, USA, 2008.

[130] Supernova Search Team Collaborations. A.G. Reiss et al: Observation Evidence from supernova for an accelerating Universe and a Cosmological constant. The Astronomical Journal, 116, 1998, pp. 1009-1038.

[131] Supernova Cosmology Project Collaboration. S. Perlmutter et al: Measurements of Omega and Lambda from 42 High-Redshift Supernova. The Astronomical Journal, 517, 1999, pp. 565-585.

[132] G.F. Smoot et al: Structure in the COBE differential microwave radiometer first year maps. The Astronomical Journal, 396, 1992, pp. L1-L5.

[133] M. Tegmark: Measuring cosmological parameters with galaxy surveys. Physics Review Letters, 79, 1997, pp. 3806-3809.

[134] W. Rindler: Relativity (Special, general and cosmological). Second Edition, Oxford University Press, Oxford, UK, 2006.

[135] D. Gross et al: Heterotic string theory (1). The free Heterotic string. Nuclear Physics B, 256, 1985, pp. $253-284$.

[136] F. Macdonald: It's official: Gravitational wave have been detected, Einstein was right. Science Alert, 11 February 2016. 


\section{Appendix 1}

\section{Illustration of the first five iterations of the construction of a deterministic Cantor set}

Referring to Figure 1 and with the aim of having a clearer representation as well as to introduce the link between measure and set, the segments in the figure were chosen as bars of fixed width and consequently representing a uniform density distributed over the support set. As the number of iterations goes to infinity, the Hausdorff dimension goes to $\ln 2 / \ln 3=0.6309297536 \simeq 0.63$.

In other words nothing remains of the initial black line of unit length except an uncountable infinite number of "Cantorian" black points (or bars) with a total length equal zero. Thus we could imagine the black line to have been replaced by a white line of the length one minus zero which is the original line of the length of the black line. Therefore we could say that we have two "Cantor" sets. The first is an infinite number of black "points" with length, i.e. measure equal zero and a Hausdorff dimension equal 0.63 apart of a topological dimension equal zero because it consists only of points at the limiting infinity.

The second set by contrast is a white line of a unit length of a Hausdorff dimension equal $1-0.63 \simeq 0.37$. It is important now to find a consistent topological dimension for this set which represents the totality of the gaps which we created in the initial black line by removing the middle third iteratively in the familiar fractal construction manner. Since the white "set" must be either nothing or an empty set, then its dimension must be negative. Formally it is minus one as is clear from von Neumann-Connes formula or it is minus infinity for the same reasons but taking into account the special situation of a one dimensional universe rather than the real infinite dimensional spacetime of Cantorian E-infinity theory [7]-[17].

\section{Appendix 2}

\section{Generalizing the classical Cantor set to two dimensions}

The two dimensional counterpart of the one dimensional triadic Cantor set is neither the two dimensional Swiss flag fractal (see Figure 7) with $D_{H}=(2)(\ln 2 / \ln 3)$ nor is it the Sierpinski carpet (see Figure 8) with $D_{H}=\ln 8 / \ln 3=3(\ln 2 / \ln 3)$ but rather the Sierpinski gasket (see Figure 9) with $D_{H}$ equal the inverse of the Hausdorff dimension of the Cantor set, namely $\ell n 3 / \ell n 2=1.584962501$. Again the white triangles correspond to empty sets with negative topological dimensions. The generic E-infinity fractal corresponding to this gasket is the two dimensional random Cantor set which is also found from the inverse of $\phi$ to give us

$1 / \phi=1+\phi=1.618033989$ which is rather close to $\ln 3 / \ln 2=1.584962501$ (see Refs. [1]-[6]).

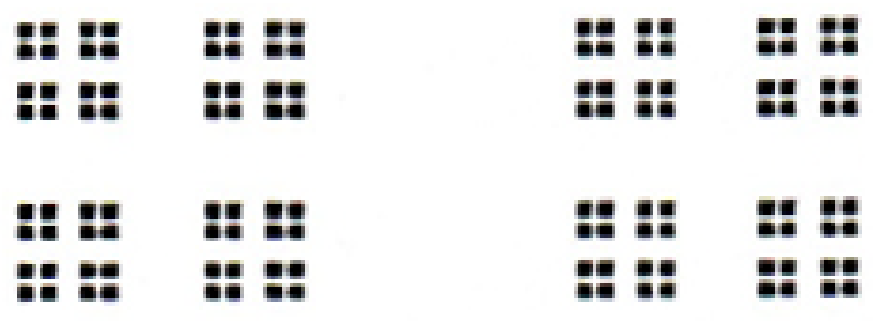

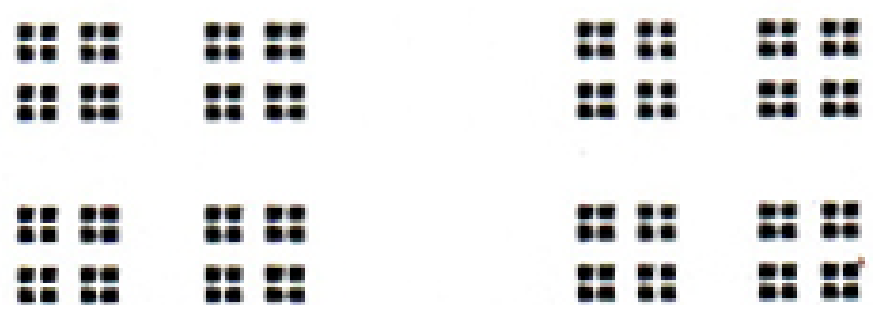

Figure 7. The Swiss flag fractal which is the cartesian product of two cantor sets. 

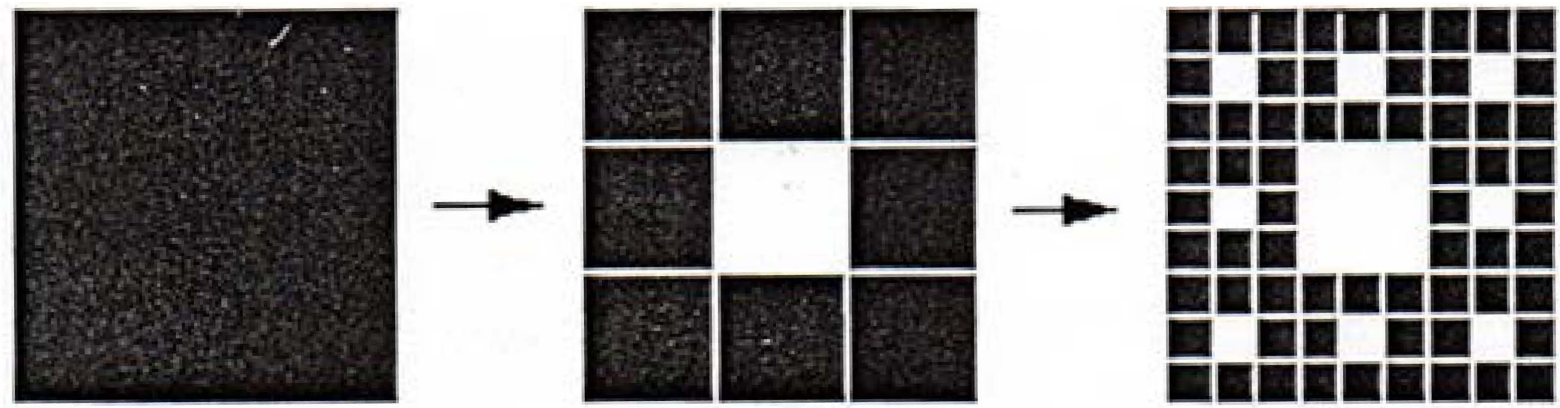

Figure 8. The Sierpinski carpet is the two dimensional version of the Menger sponge. The generic case is found for a random fractal to that found from the bijection formula of E-infinity theory $d_{c}^{(n)}=(1 / \phi)^{n-1}$ by setting $n=2$ and finding that $d_{c}^{(2)}=(1 / \phi)=1.618033989$.
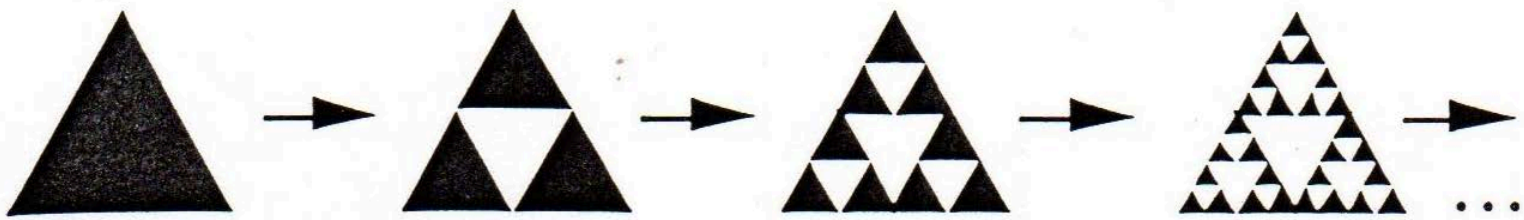

Figure 9. The Sierpinski gasket as the right way to lift the one dimensional triadic Cantor set $D_{(H)}=\ln 2 / \ln 3$ to two dimensions. The Hausdorff dimension in this case is the inverse of $\ln 2 / \ln 3$, namely $\ln 3 / \ln 2$. Thus it obeys the dimensional function of von Neumann-Connes (see Ref. [107]).

\section{Appendix 3}

\section{Generalizing the classical Cantor set to three dimensions}

Referring to Figure 10 and Figure 11 we can say the following:

The generic generalization of Cantor's triadic set to 3 dimensions is neither that shown in Figure 10 nor is the Menger sponge of Figure 11 which is a generalization of the Sierpinski carpet shown in Figure 8. On the other hand a generalization of a random Cantor set of the Mauldin-Williams type, i.e. is easily achieved for any dimension using von Neumann-Connes dimensional function or equivalently the bijection formula of E-infinity theory $d_{c}^{(n)}=(1 / \phi)^{n-1}$ where $n$ is the topological dimension. That way a "generic" random Menger sponge dimension should be $D(H)=(1 / \phi)^{3-1}=2+\phi=2.618033989$ and in four dimensions it is $D(H)=(1 / \phi)^{3}=4+\phi^{3}$ (for details see Refs. [1]-[6] and Ref. [10]).

\section{Appendix 4}

\section{Equivalence relations in mathematics and physics}

The interest of the present work in the pure mathematics and mathematical logic of equivalence relations stem from numerous expected and less expected connections to theoretical and mathematical physics [1]-[111]. For instance we know that equivalence relations is a binary relation that is reflexive as well as symmetric and transfinite, all simultaneously [108]. From that we can deduce that this equivalence relation will result in a partition of a set of equivalence classes. In other words, it results in a quotient set of a quotient space. A generic quotient space on the other hand is the $x$ space representing Penrose fractal tiling universe [7]-[17]. In turn this space is a prototype of A. Connes' noncommutative geometry as well as a realization of E-infinity Cantorian spacetime with the same dimensional function [75]. In other words, it is akin to a similarity classifier with a golden mean [109] and could be drawn into understanding the similarity of Einstein's formula $E=m c^{2}$, as well as the Planck radiation expression $E=h f$ and possibly Newton's second law $F=m a$ as well as the similarity of Newton's kinetic energy $E=1 / 2 m v^{2}$ and our dissected Einstein formula $E=\left(m c^{2} / 22\right)+m c^{2}(21 / 22)=m c^{2}[53]-[59]$. 


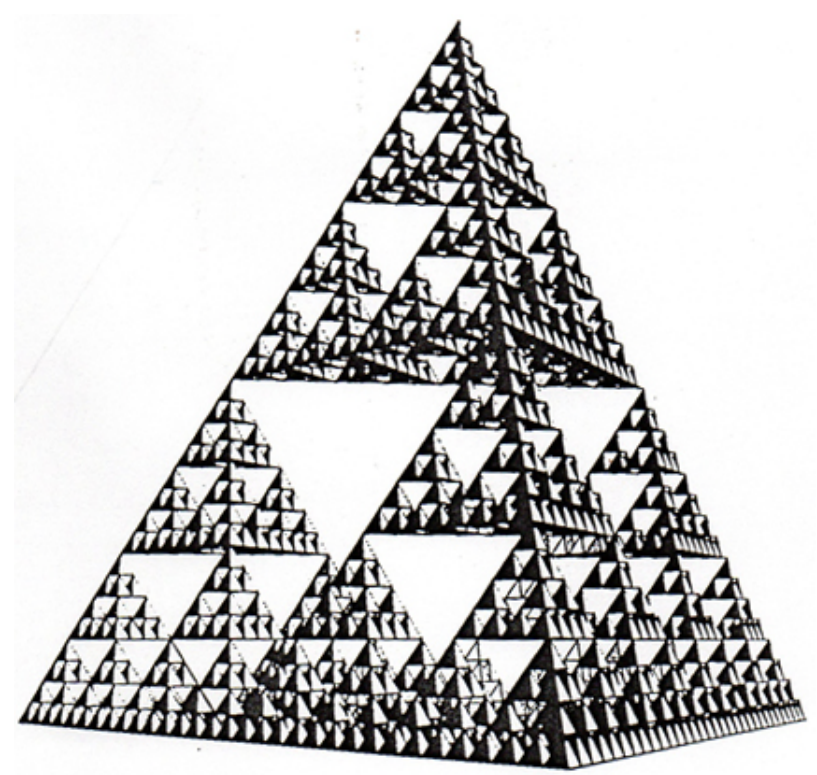

Figure 10. A three dimensional version of the Sierpinski gasket. Its fractal dimension $\log 4 / \log 2=2$ has an integer value (2), albeit smaller than the dimension of the supporting space (3) (see Refs. [1]-[5]).

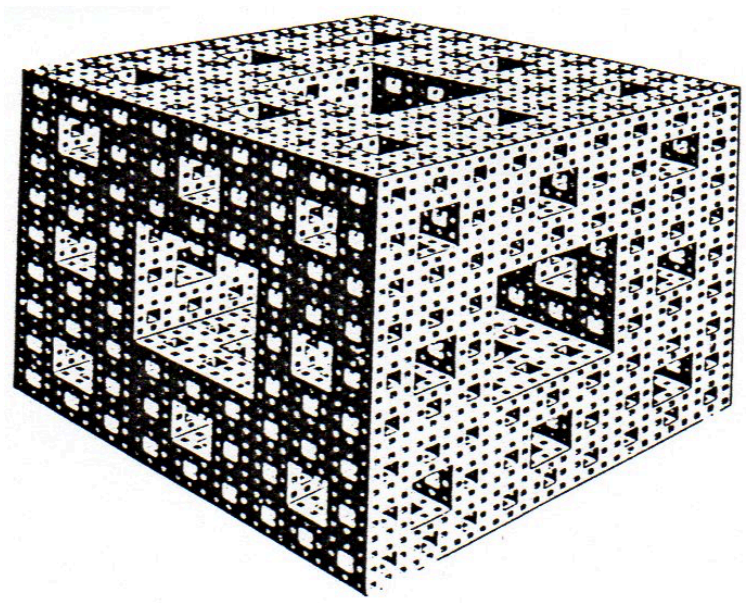

Menger sponge $D=\log 20 / \log 3 \cong 2.73$

Figure 11. The Menger sponge although it is not the real generalization of a Cantor set to three dimensions has a Hausdorff dimension quite close to the random three dimensional Cantor set $\left(D=(1 / \phi)^{2} \simeq 2.618033989\right)$. It is used in many applications including modelling three dimensional fractal space.

\section{Appendix 5}

\section{Geodolian role in the triality of logic}

This short appendix is intended to show how fuzziness enters into physics via logic. Let us start with classical "ordinary logic". Here we have zero and one which may be taken to correspond to yes or nor or equivalently, right and wrong. On the other hand Gödel's theorem establishes at a minimum the possibility of a three valued logic corresponding to the above, i.e. yes and not plus a third possibility of undecidability which we may call in plain English "do not know". The E-infinity set theoretical realization of this triality is aptly set theory, namely the zero set and the empty set. It is remarkable that this seemingly unconventional logic leads to a disarmingly simple and intuitive realization, namely yes for sets and do not know for the zero set. The first and the second 
identifications are obvious and self evident. By contrast the third possibility may need some further elaboration. This is because undecidability has an element of fuzziness while a zero set is crisp, i.e. sharp. Our response to this point is that a zero set is not zero but a set containing zeros as "elements". When the zeros disappear completely we are still left with a set, albeit an empty set. Consequently a zero set is not as sharp as a zero but represents the logical location of the zero being the border separating positiveness and negativeness on a number world line. Seen from this viewpoint it is thus the dynamics created by set, empty set and zero sets that represent a true fuzzy dynamics and incidentally it also corresponds to G. Ord's anti-Bernulli processes, i.e. 1, 0, -1 which gives us quantum mechanics while the Bernulli processes gives us a mere diffusion.

\section{Appendix 6}

\section{Important remarks on Schrödinger's equation and special relativity}

Fusing Schrödinger's equation, i.e. quantum mechanics with $E=m c^{2}$, i.e. special relativity does not result in a quantum gravity theory but rather in a Dirac equation, i.e. a Schrödinger equation without its nonlocal character. Similarly a quantum gravity theory is not a complete unification of all fundamental forces because for instance it leaves out electromagnetism. For this reason one has to be quite critical and on guard from undue generalization to avoid later misconceptions arising from reading too much into partial unification of the five different fundamental interactions.

\section{Appendix 7}

\section{Remarks on the dependence of the empty Cantor set on the dimensionality}

Let us consider a one dimensional random Cantor set. The topological dimension is clearly zero and its Hausdorff dimension is $\phi$. On the other hand what is left from this set is a complimentary set with some contradictory characteristics. This set is made from the gaps created in the iterative process. It is completely empty gaps and one would think that its Hausdorff dimension should be zero. However it is not. Its Hausdorff dimension is the complement of $\phi$, namely $1-\phi=\phi^{2}$ which corresponds to a topological dimension equal -1 only while we would have expected that the corresponding dimension is $-\infty$. This contradiction arises only because we limited generality to one topological dimension of a one dimensional world line. This contradiction does not exist in the Cantorian theory of spacetime where the covering equation is truly general and given by either the von Neumann-Connes dimensional function [107]

$$
D=a+b \phi, a, b \in Z \text { and } \phi=(\sqrt{5}-1) / 2
$$

or the bijection equation of E-infinity theory [10] [13]

$$
d_{c}^{(n)}=(1 / \phi)^{n-1}
$$

where $-\infty \leq n \leq \infty$ i.e. $n$ runs from $-\infty$ to $+\infty$.

\section{Appendix 8}

An attempt towards a simple intuitive elucidation and elementary derivation of $E=\mathrm{mc}^{2} / 22$ as an Eigenvalue problem

We have here a remarkable Eigenvalue problem. It is $E=m c^{2}$ which almost everybody knows because it is arguably the most famous formula in the history of physics. Energy in this way is comparable to the buckling load in an Eigenvalue stability problem. As we know it is $P=E I \pi^{2} / l^{2}$ for a compressed simply supported strut [114]. Except for the numerical value 9.86960 the two formulas are comparable. In Einstein's equation the number is simply unity and in the buckling equation the number is almost 10. Now when we solve the buckling problem using Rayleigh-Ritz trivial function or some other similar approximation, one normally finds a higher value, say 12 and then as one increased the number of finite elements which mean we increased the degrees of freedom then we come nearer and nearer to the exact value. Now Einstein's formula was obtained from a one degree of freedom theory [114]. In high energy physics we count as a degree of freedom all so called messenger particles [46]. That is all what you need to know from physics. At the time when Einstein drove his equations, i.e. the Eigenvalue the world knew of a single messenger particle only which is the photon. A photon is a small 
elementary quantum particle which is seen as light but we can experiment with it in the meantime and we can have this little bit of indivisible particle called photon. As time went by we have now almost finished refining our standard model and we know what Einstein did not know at the time, namely that there is something called quantum mechanics and that the standard model has 12 photon-like messenger particles and not only a single photon particle. Thus topologically or geometrically or mechanically, whatever one would like to say, the model which Einstein tinkered with and got his formula as an Eigenvalue with one degree of freedom has in reality 12 degrees of freedom. Needless to say, Einstein did not solve his problem as an Eigenvalue problem and did not look upon his analysis as such. This Eigenvalue entered into physics of the photon only when Paul Dirac incorporated Einstein's special relativity into Schrodinger's equation which resulted in this formula of Einstein being one of the energy levels Eigenvalues [46]. Schrodinger motivated that by a famous paper in German called "Quantization as an Eigenvalue" [46].

All that would have remained unimportant or at a maximum of minor academic value if it were not for the following.

When they tested the equation of Einstein in the laboratory within a room not larger than a sitting room, they found experimentally that it represents the energy density expected. Yes Einstein equation of converting mass into pure energy although mostly theoretical, never the less represented a practically correct maximal energy density. The shock came some 15 years or so ago when in repeated experiments using the entire universe as a laboratory cosmologists attempted to calculate the energy density of the entire universe using super nova events and as revealed by the WMAP experiment and confirmed by Planck and other cosmic measurements. They found approximately $4.5 \%$ only of the expected $100 \%$ density [60]. From this point of view Einstein's maximal energy is the $100 \%$ and the numerical factor 1 represents this $100 \%$ just like in the buckling problem, the numerical value of almost 10 represents the exact solution which means the $100 \%$ correct solution. However in the case of Einstein's formula we are missing 95.5\%. This is not a rounding error nor inaccuracy of measurement or error caused by using approximate solutions. The $95.5 \%$ error is a disaster by all standards. The experimental measurements are in the meantime beyond doubt and were awarded in 2011 the Nobel Prize for physics or cosmology to three scientists [60].

If the analogy between the buckling load and Einstein's formula as an Eigenvalue holds, then the discrepancy could be explained as restricting the system and forcing the energy density of Einstein to be much higher than the real energy density is. The reason for the small amount of energy of $4.5 \%$ they found was suspected by the author immediately when he heard and understood the problem that it might be related to the 11 extra degrees of freedom which Einstein did not take into account. Einstein was limited by history and his training. He did not know at the time of the experimental facts and quantum mechanics was not invented yet and CERN did not yet exist. Moreover, when he got to know quantum mechanics he was not ready to accept it and the fight between him on the one side and Bohr, Heisenberg and Schrodinger on the other is well known in the history of science [46]. So as I said, if this is correct and the author believes it to be correct then the factor 1 in Einstein's formula should be in reality $1 / 22$ to get the correct observed results of cosmic measurements of WMAP and the rest.

Now the author solved this problem using quite sophisticated ad complex mathematical physics as shown for example in the present paper. However the author believes that a simpler and more intuitive explanation could lead us to the correct result. The author had such a simple idea and he even presented it conferences but normally he published the sophisticated mathematics.

If there is a simple watertight solution based on similarity and number of degrees of freedom then this must be based on the similarity between $E$ (kinetic) $=1 / 2 m v^{2}$ and $E($ Einstein $)=m c^{2}$. Now it is not difficult to see that the $12-1=11$ degrees of freedom not included in $E=m c^{2}$ could be thought of as a scaling $\lambda_{o}=1 / 11$ and inserting in Newton's kinetic energy while letting $v \rightarrow c$ one finds $E=(1 / 2)(1 / 1)(m)(v \rightarrow c)^{2}$. This result agrees not only with cosmic measurements but also with the results obtained using quite sophisticated mathematics similar to what was done in the main body of the present paper. In short we need to make the present simple scaling argument mathematically watertight because the author feels it is not yet and wrote this short appendix mainly in the hope that some of the readers with more mathematical skills could find a mathematical way to argue the case. 